\title{
1 A Vibrio cholerae Core Genome Multilocus Sequence Typing Scheme to Facilitate the
}

\section{Epidemiological Study of Cholera}

4 Kevin Y. H. Liang, ${ }^{\text {a }}$ Fabini D. Orata, ${ }^{a}$ Mohammad Tarequl Islam, ${ }^{a}$ Tania Nasreen, ${ }^{\text {a }}$ Munirul

5 Alam, ${ }^{\mathrm{b}}$ Cheryl L. Tarr, ${ }^{\mathrm{c}}$ Yann F. Boucher ${ }^{\mathrm{a}}$

6

7 aDepartment of Biological Sciences, University of Alberta, Edmonton, Alberta, Canada

8 bInfectious Diseases Division, International Centre for Diarrhoeal Disease Research,

9 Dhaka, Bangladesh

10 'Enteric Diseases Laboratory Branch, Centers for Disease Control and Prevention, Atlanta,

11 Georgia, USA

12

13 Running title: $V$. cholerae Core Genome Multilocus Sequence Typing

14

$15 \quad$ \#Address correspondence to Yann F. Boucher, yboucher@ualberta.ca 


\section{ABSTRACT}

17 Core genome multilocus sequence typing (cgMLST) has gained popularity in recent years in

18 epidemiological research and subspecies level classification. cgMLST retains the intuitive nature

19 of traditional MLST but offers much greater resolution by utilizing significantly larger portions

20 of the genome. Here, we introduce a cgMLST scheme for Vibrio cholerae, a bacterium abundant

21 in marine and freshwater environments and the etiologic agent of cholera. A set of 2,443 core

22 genes ubiquitous in $V$. cholerae were used to analyze a comprehensive dataset of 1,262 clinical

23 and environmental strains collected from 52 countries, including 65 newly sequenced genomes

24 in this study. We established a sublineage threshold based on 133 allelic differences that creates

25 clusters nearly identical to traditional MLST types, providing backwards compatibility to new

26 cgMLST classifications. We also defined an outbreak threshold based on seven allelic

27 differences that is capable of identifying strains from the same outbreak and closely related

28 isolates which could give clues on outbreak origin. Using cgMLST, we confirmed the South

29 Asian origin of modern epidemics and identified clustering affinity among sublineages of

30 environmental isolates from the same geographic origin. Advantages of this method are

31 highlighted by direct comparison with existing classification methods, such as MLST and single

32 nucleotide polymorphism-based methods. cgMLST outperforms all existing methods in terms of

33 resolution, standardization, and ease-of-use. We anticipate this scheme will serve as a basis for a

34 universally applicable and standardized classification system for $V$. cholerae research and

35 epidemiological surveillance in the future. This cgMLST scheme is publicly available on

36 PubMLST (https://pubmlst.org/vcholerae/). 


\section{IMPORTANCE}

38 Toxigenic Vibrio cholerae of the $\mathrm{O} 1$ and $\mathrm{O} 139$ serogroups are the causative agent of cholera, an

39 acute diarrheal disease that plagued the world for centuries, if not millennia. Here, we introduce

40 a core genome multilocus sequence typing (cgMLST) scheme for $V$. cholerae. Using cgMLST,

41 we established an outbreak threshold that can efficiently identify outbreak related strains and

42 potential sources of introduction. We also defined a sublineage threshold that is similar to

43 traditional MLST sequence type which will provide context to this new typing method by

44 relating it to previous MLST results. cgMLST outperforms all existing methods in terms of

45 resolution, standardization, and ease-of-use, making this scheme the most suitable method for $V$.

46 cholerae typing and surveillance worldwide.

47

\section{KEYWORDS}

49 Vibrio cholerae, cholera, whole genome sequencing, core genome, multilocus sequence typing,

50 cgMLST, gene-by-gene approach, epidemiological surveillance 


\section{INTRODUCTION}

52 Cholera is transmitted in a fecal-oral route mostly by contaminated food or water $(1,2)$. The case

53 fatality rate (CFR) of this disease can be up to $50 \%$ without treatment, but with proper medical

54 care, the CFR is usually less than $1 \%(2,3)$. In developed countries with proper water treatment

55 facilities, cholera is practically non-existent aside from imported cases. Unfortunately, this

56 cannot be said for many developing countries lacking this infrastructure, where cholera has been

57 endemic for centuries such as in parts of South Asia (4). As it is also difficult to eradicate cholera

58 (5), this disease often becomes endemic in regions where it has been introduced, for example in

59 Latin America in 1991 (6, 7), Haiti in 2010 (8), and Yemen in 2016 (9). It is estimated that there

60 are over a million cholera cases each year resulting in tens of thousands of deaths worldwide (10).

61 Being an indicator of healthcare and socio-economic disparities $(11,12)$, this disease is often

62 under-reported due to its negative influence on tourism as it implies poor water quality (13).

63 Together with the lack of a universally applicable and standardized classification method,

64 outbreak surveillance and source attribution is often challenging $(1,8)$. The Haiti outbreak for

65 example, due to these limitations, required extensive genomic and epidemiological research

66 since the beginning of the outbreak to determine the source of introduction, which was not

67 confirmed until August 2011 even though cholera broke out in July 2010 (8, 14-17).

68 A typing method for use in global surveillance of pandemic causing pathogens such as $V$.

69 cholerae should be efficient and easy to use, with the potential to be applied to all $V$. cholerae

70 strains around the world. Therefore, it must have the capacity to analyze thousands of genomes

71 efficiently and new genomes should be easily typed as they get sequenced. As all cholera

72 outbreaks are caused by a single lineage of $V$. cholerae, the pandemic generating/phylocore

73 genome (PG) lineage, which includes the $7^{\text {th }}$ pandemic El Tor, El Tor sister, El Tor progenitor, 
74 Classical, and Classical sister clades $(5,18,19)$, this method should also be able to differentiate

75 isolates at a fine scale and separate outbreaks caused by genetically similar strains. Such a

76 method will help create a comprehensive database with detailed epidemiological data that will

77 allow for the analysis of future outbreak strains in a global context and guide subsequent

78 epidemiological analyses. Different methods for subspecies level classification and outbreak

79 surveillance have been developed for $V$. cholerae. These methods include serotyping, multilocus

80 sequence typing (MLST) (20, 21), multilocus variable number of tandem repeats (VNTR)

81 analysis (MLVA) $(22,23)$, and single nucleotide polymorphism (SNP)-based approaches (14).

82 Despite the popularity of these methods, there are important limitations to each.

83 Serotyping based on the presence of cell surface O-antigens is one of the earliest attempts

84 at subspecies level classification of $V$. cholerae. There are now over 200 serogroups of $V$.

85 cholerae identified; however, only the toxigenic members of the $\mathrm{O} 1$ and $\mathrm{O} 139$ serogroups have

86 been found to be responsible for all major documented epidemics and pandemics $(24,25)$.

87 Serogroup O1 can be further divided into two biotypes (El Tor and Classical) and three serotypes

88 (Inaba, Hikojima, and Ogawa) (2). The lack of resolution within the epidemic strains and the

89 possibility of serogroup conversion (26) limits the use of serotyping in epidemiological studies.

91 seven well-defined housekeeping genes (27). MLST was used to study a number of cholera

92 outbreaks and allowed the descriptions of its general population structure $(28,29)$. It is

93 reproducible and provides reliable results; however, it is unable to differentiate between closely

94 related strains which limits its use in outbreak surveillance $(30,31)$. In addition, direct

95 comparisons between different MLST schemes are difficult, as different schemes utilize different

96 housekeeping genes. 
MLVA utilizes VNTR regions, which are under less selective pressure than housekeeping genes. This method therefore provides greater resolution than MLST for some bacterial species

$99(32,33)$. However, due to their rapid mutation rate, VNTR regions are more affected by

100 homoplasy where two isolates may share the same MLVA profile due to convergent mutation

101 and not by vertical descent (34). As a result, MLVA may produce clusters that do not necessarily

102 reflect phylogenetic relationships (35). Two common PCR-based methods exist for the typing of

103 VNTR regions, but each have significant limitations (36). The first method is multiplex PCR

104 which can analyze all loci at once, but it is impossible to determine which bands correspond to

105 which loci; therefore, this method only produces a banding pattern for strain identification,

106 which makes it difficult to standardize and communicate results. The second method is the

107 separate amplification of VNTR regions but determining the number of repeats based on

108 amplicon size information alone is difficult if the difference in size is not large enough. In

109 addition, different types of mutations that do not necessarily change the number of repeats can

110 cause a change in amplicon size. Sequencing is needed to confirm MLVA profiles, but repeat

111 regions increase the chances of sequencing errors (37). Due to these limitations, stringent quality

112 control is required for reliable MLVA analysis (38).

113 SNP-based analysis is one of the most common whole genome-based methods currently

114 being used and was applied to various outbreaks $(14,39,40)$. It relies on the identification of

115 conserved SNPs in strains of interest using next-generation sequence reads or assembled

116 genomes. The number of SNPs can then be related to the evolutionary distance between isolates.

117 SNP-based analysis provides reliable results with sufficient resolution for epidemiological

118 studies, but it is sensitive to horizontal gene transfer and recombination events, as each event will

119 result in many SNPs being created. The number of SNPs between two strains, therefore, does not 
120 necessarily reflect true phylogenetic relationship. SNPs found in recombinogenic regions should

121 therefore be removed which, depending on the organism of interest, can be anywhere from $30 \%$

122 to $97 \%$ of SNPs identified $(41,42)$. Since recombination and horizontal gene transfer events are

123 common within $V$. cholerae (43-45) and between the species and its close relatives $(46,47)$,

124 SNP-based methods, although suitable in individual epidemiological studies, will be difficult to

125 serve as a universal classification method for $V$. cholerae.

126 Core genome MLST (cgMLST), also known as the gene-by-gene approach, overcomes

127 the various limitations of the previously mentioned subtyping methods and was established to

128 serve as a universally applicable standardized typing scheme. Similar to MLST, cgMLST relies

129 on individual gene sequences to differentiate between closely related strains; however, instead of

130 using only six to seven housekeeping genes, cgMLST utilizes hundreds to thousands of core

131 genes, which are commonly found in all strains of a species. By utilizing a much larger portion

132 of the genome, cgMLST provides superior resolution compared to traditional MLST schemes.

133 By combining the expandable and standardized classification method that made traditional

134 MLST favourable with the resolution of whole genome-based methods, cgMLST is becoming

135 more popular in epidemiological and ecological studies (48-54). This method has the added

136 advantage of backwards compatibility with all MLST schemes. This means that it is possible to

137 determine MLST profiles of any isolates based on their cgMLST profiles, since cgMLST would

138 include all housekeeping genes by definition. This allows for a 1:1 mapping of any previously

139 established MLST scheme to the cgMLST scheme, helping consolidate already existing

140 classification information.

Another major benefit of cgMLST is that, much like traditional MLST methods, it is

142 possible to establish different clustering thresholds to define important groups. Clonal complexes 
143 are examples of clustering thresholds established by MLST schemes, where each clonal complex

144 corresponds to a cluster of isolates that share at most one allelic difference across all seven genes

145 sequenced. Some important clonal complexes were shown to correspond to either groups

146 established by a previous typing method (55) or major outbreak strains (56). However, cgMLST

147 offers even greater flexibility than MLST in this regard given the number of loci considered.

148 With large clustering thresholds, it is possible to identify lineage- or even sublineage-level

149 differences to study large scale patterns and answer broader ecological questions. Furthermore,

150 with smaller clustering thresholds where groups are created based on the sharing of a larger

151 number of alleles, it is possible to identify very closely related strains useful in epidemiological

152 studies. The benefits of defining clustering thresholds through cgMLST have already been

153 demonstrated in other human pathogens, such as Brucella melitensis (52), Campylobacter jejuni

154 (51), Clostridium difficile (53), Enterococcus faecium (50), and Listeria monocytogenes (49).

155 In this study, we introduce a cgMLST scheme for the genome-wide typing of $V$. cholerae

156 and demonstrate its universality and efficacy by applying it to known cholera outbreaks around

157 the world. The advantages of cgMLST are presented by comparing the scheme with previously

158 established classification methods. Additionally, we have produced a 1:1 mapping of the

159 cgMLST scheme against two previously established MLST schemes for $V$. cholerae (20, 21),

160 allowing for the consolidation of existing classification information. The cgMLST scheme,

161 genome sequences used in this study, and relevant epidemiological information are publicly

162 available on PubMLST (https://pubmlst.org/vcholerae/), which allows for the automatic

163 annotation and subsequent analyses of hundreds of newly uploaded $V$. cholerae genomes in a

164 global context. This increase in efficiency, standardizability, and resolution compared to current

165 methods make cgMLST the most suitable classification scheme for large scale $V$. cholerae 
surveillance. By applying this scheme to our collection of over 1,200 isolates collected around the world, it was possible to establish outbreak and sublineage thresholds which not only allowed us to validate the South Asian origin of many modern epidemics as proposed in previous studies

$169(5,57,58)$ but also identify clustering affinity among environmental strains, where isolates from

170 the same sublineage are also likely from the same geographic region. This pattern is not seen in

171 clinical isolates, as human hosts readily carry them over large geographical distances.

173 RESULTS AND DISCUSSION

174 A high-resolution typing scheme for pandemic $V$. cholerae

175 The highest level of resolution of any cgMLST scheme is defined by core genome sequence

176 types (cgSTs), where a unique cgST represents a unique allelic profile. Isolates that belong to the

177 same cgST are expected to be phylogenetically very closely related, as although they may not

178 have the exact genomic sequence, they do have the same sequence for all 2,443 core gene loci

179 used in cgMLST. We identified a total of 1,026 cgSTs from 1,262 genomes collected from 52

180 countries. Even with our extensive dataset, we have yet to sample anywhere close to the total

181 predicted cgST diversity for the global V.cholerae population (Fig. S1). All isolates were given

182 at least one cgST designation and up to two MLST sequence type (ST) designations based on

183 two previously established MLST schemes $(20,21)$ (Table S1). MLST STs are defined based on

184 the unique combination of all loci of a particular MLST scheme, which ideally uses six to seven

185 well-defined housekeeping genes. Only 12 STs are exclusively present in the $7^{\text {th }}$ pandemic El

186 Tor lineage identified using traditional MLST $(20,21)$, whereas 560 cgSTs are uniquely present

187 in this group based on cgMLST (Table S1). As the E1 Tor lineage is responsible for most cholera

188 outbreaks around the world since the beginning of the $7^{\text {th }}$ pandemic (59), this superior ability to 
189 resolve between closely related strains in the $7^{\text {th }}$ pandemic El Tor lineage makes cgMLST more

190 suitable in outbreak surveillance than traditional MLST.

192 Backwards compatibility with previous subspecies classification methods

193 Much like how cgSTs are important in studying closely related strains typical in outbreaks, it is

194 also important in establishing a standardized nomenclature at a higher level to answer broader

195 ecological questions. Here, we propose a sublineage definition for $V$. cholerae based on cgMLST.

196 Pairwise allelic differences calculated between all isolates show three major peaks (Fig.

197 1A). The first peak ends at 40 allelic differences, and the second peak ends at 133 allelic

198 differences (Fig. 1B). The last peak begins at 2,200 allelic differences (Fig. 1A), which is

199 expected due to mutational saturation (i.e., every single allele in the scheme is different between

200 the two distantly related strains being compared). Both breaks (i.e., 40 or 133 allelic differences)

201 could represent a potential sublineage delineation. To choose between the two thresholds, the

202 clustering efficiency is measured by calculating the Dunn Index (DI) (60) (see Materials and

203 Methods). Since cluster distances are measured by allelic differences, the network with the best

204 clustering efficiency (i.e., the highest DI) will also produce clusters that best represent biological

205 relationships, as isolates are more closely related to themselves than to isolates from other

206 clusters. A DI was calculated for each clustering threshold in the range of 1 to 1,000 allelic

207 differences with 100 bootstrap replicates (Fig. 2). As the clustering threshold defines the

208 maximum number of allelic differences within a cluster, the smaller the threshold, the more

209 closely related the isolates are within a cluster. It is clear that DIs in the range of 0 to 50 allelic

210 differences are significantly lower than the DIs in the range of 100 to 350 allelic differences,

211 with 133 being a clear local maximum. Since 133 allelic differences has the best clustering 
212 efficiency and also represents a natural break where most isolate pairs have either less than or

213 much greater number of allelic differences (Fig. 1B), it was therefore chosen as the sublineage

214 threshold.

215 Because cgMLST includes all housekeeping genes, information from the two MLST

216 schemes previously developed for $V$. cholerae $(20,21)$ can now be consolidated with the

217 cgMLST scheme by creating a 1:1 cgMLST to MLST map. To evaluate the similarities between

218 the sublineage threshold and the MLST schemes, we created a minimum spanning tree (MST)

219 for all Bangladesh isolates $(n=255)$ showing only edges with 133 allelic differences or fewer

220 (Fig. 3A and Fig. S2). Each cluster therefore represents a single sublineage. Bangladesh was

221 chosen to compare cgMLST and MLST as it is the most extensively sampled country both in

222 terms of clinical and environmental isolates in our dataset. Using this dataset, the chosen

223 sublineage threshold produces clusters that closely resemble ST clustering from traditional

224 MLST. Based on the 2013 MLST scheme (20), each sublineage corresponds to exactly one ST

225 (Fig. S2), whereas there is only one sublineage that contains two STs based on the 2016 MLST

226 scheme (21) (Fig. 3A). All but two isolates belong to ST1; N16961 and A19 belong to ST290,

227 which differs from ST1 at only one of seven MLST loci (Table S2). The reason these two

228 isolates are of a different MLST ST could only be partly explained; they were isolated at an

229 earlier time point (1970s near the start of the $7^{\text {th }}$ pandemic (61)) than most of the remaining

230 isolates, which were isolated from 1991 onwards (Table S1).

231 It is impossible to visually evaluate similarities between two MSTs with over 1,200 nodes

232 each simply due to the sheer volume of data. The Adjusted Rand Index (ARI) was therefore used

233 as a metric to determine network similarities (62) (see Materials and Methods). In order to

234 determine whether the sublineage threshold (i.e., 133 allelic differences) is indeed the best match 
235 to traditional MLST schemes, we chose 11 clustering thresholds distributed across the range of 1

236 to 1,000 allelic differences (Fig. 2) to compare with the MLST schemes. These additional

237 thresholds are chosen as they have a relatively high DI compared to their immediate neighbours.

238 More data points were chosen in the range of 105 to 330 allelic differences, as it was expected

239 that thresholds in this range will best match the traditional MLST schemes. Interestingly, all

240 thresholds in that range had comparable ARIs when compared to both the $2016(21)$ and the

2412013 MLST schemes (20) (Fig. 3B and Fig. S3), indicating that all of them, including the

242 sublineage threshold, produce clusters similar to the MLST scheme. This would suggest that

243 there can be a large range of diversity within a single MLST ST where isolates can have

244 anywhere from 0 (i.e., have the same cgST) to 330 allelic differences. Although clustering

245 thresholds between 105 to 330 allelic differences produce similar clusters to a traditional MLST

246 scheme, the sublineage threshold of 133 allelic differences was chosen as it has the best

247 clustering efficiency (Fig. 2) and it represents a natural breakpoint in the currently sampled 248 population (Fig. 1B).

A phylogenetic tree of 1,146 isolates was used to assess the phylogenetic support of the

250 sublineage threshold across different $V$. cholerae strains (Fig. 4). This tree includes all $V$.

251 cholerae isolates within our dataset with the exception of the recently published 116 clinical

252 isolates from the Yemen cholera outbreak (9), which all belongs to the $7^{\text {th }}$ pandemic El Tor

253 lineage. The strains within the PG lineage are closely related with little genetic variation. These

254 lineages are therefore collapsed in the phylogenetic tree as the relationships between them are

255 not well resolved. All sublineages formed monophyletic clades, although in some cases the most

256 basal branch is of a different sublineage (e.g., V. cholerae strains T5 or 506315) creating

257 paraphyletic clades. Ideally, each sublineage would correspond to exactly one full monophyletic 
clade. The reason this is not seen is likely the lack of sampling, leading to the grouping of

259 relatively distantly related isolates together in the same clade. Further sampling will likely

260 resolve these cases into two separate monophyletic clades. Out of 1,262 isolates, we identified

261291 sublineages, 19 of which belong exclusively to the PG lineage and 223 are singletons. Much

262 like cgSTs, the rarefaction curve also indicates that the total sublineage diversity of $V$. cholerae

263 is far from being sampled (Fig. S1).

264 The sublineage concept has been applied to numerous pathogens and as such were each

265 defined differently depending on the pathogen in question. Some have defined sublineages based

266 on natural breaks in genetic similarities (49), while others may use sublineage to refer

267 specifically to traditional MLST STs (63) or even finer level of resolution below the MLST ST

268 level based on whole genome analyses (64). There is, however, one unifying feature of all

269 sublineage definitions - that they all refer to monophyletic clades. Sublineages are defined in this

270 study based on natural breaks in allelic differences calculated from cgMLST profiles and were

271 put into context by comparing with two traditional MLST schemes. We have shown that our

272 definition of sublineage results in monophyletic clades but also corresponds to any traditional

273 MLST ST designation (Fig. 4). This sublineage definition will therefore play a crucial role in

274 consolidating information from all previous MLST analyses.

275

\section{A universal South Asian origin for modern cholera outbreaks}

277 With the continual improvement of next-generation sequencing techniques, whole genome

278 sequencing is expected to become a standard practice or even the first identification tool used in

279 clinical and epidemiological studies. It is therefore critical to develop a rapid typing scheme for

280 genome sequence data that has the power to inform us about the relationship of a novel isolate 
with known strains. This is done here by defining what we term an 'outbreak threshold' based on cgMLST, which can identify outbreak related strains and potential sources of introduction. The outbreak threshold is expected to be less than 40 allelic differences as isolates from the same

284 outbreak are very closely related $(9,14)$. There is a minor discontinuity at seven allelic differences where most isolate pairs have either less or more than this number of allelic

286 differences (Fig. 1B). Looking at the DI, the local maximum in the range of 0 to 50 occurs at 287 seven allelic differences as well (Fig. 2), making this cutoff a likely candidate for an outbreak 288 threshold. When applying the outbreak threshold to a full dataset containing all sequenced $V$. 289 cholerae genomes meeting the minimum quality threshold, major clusters were examined to 290 evaluate the ability of cgMLST to identify strains that are part of the same outbreak.

291 One of the major outbreak clusters identified, with no prior information required, 292 contains the Haiti and the Yemen outbreaks, which are the two best documented cholera 293 outbreaks in modern history $(8,9,14,16,65)$. Isolates collected from these outbreaks form a 294 single cluster with the Dominican Republic, Eurasian (India, Russia, Nepal, and Ukraine), and 295 African (Tanzania, Kenya, and Somalia) isolates (Fig. 5A). The Dominican Republic isolates are 296 closely related to the Haiti outbreak strains. Given the close proximity of the two countries, co297 located on the island of Hispaniola, it was expected that isolates from Haiti would eventually 298 spread to the Dominican Republic (14). The $7^{\text {th }}$ pandemic El Tor lineage spread across the world 299 from South Asia in three separate waves (61). The third wave, being the most recent distribution 300 event, has been claimed to be responsible for the outbreaks in Haiti and Yemen (9). It is 301 therefore not surprising to see Haiti and Yemen form a single cluster with India (i.e., South Asia) 302 at its center. Nepal is the known source of introduction for the Haiti outbreak in 2010 (16), and 
303 comparison with over 1,200 V. cholerae isolates from all over the world still shows the Nepalese

304 isolates as the closest relatives to the Haitian isolates (Fig. 5A).

305 Cholera is still endemic in Africa (10) and caused several major reported outbreaks in

306 different countries over last few decades (66) including Mozambique (23, 67) and Zimbabwe

307 (68). Another major outbreak cluster groups most of the Mozambique isolates together with two

308 Zimbabwe isolates (strains CP1038(11) and 2011EL-1137) and one USA isolate (2009V-1116)

309 (Fig. 5B). Based on cgMLST, it is evident that the two Zimbabwe isolates are closely related to

310 the Mozambique isolates, differing at only four or less alleles. The close proximity of the two

311 countries suggests that these are likely travel-associated cases. Although outbreaks involving the

312 Mozambique isolates (23) and the Zimbabwe isolates $(58,69)$ have been independently studied,

313 the link between these isolates have not been shown before. Global cgMLST analysis is therefore

314 an invaluable tool as it allows for the identification of links between independent studies.

315 However, with only two Zimbabwe isolates in the dataset, additional sampling in this region is

316 required to understand the epidemiology of this outbreak. According to the NCBI BioSample

317 database, strain 2009V-1116 was collected by the Centers for Disease Control and Prevention in

3182009 and is associated with travel to Pakistan. Since the $7^{\text {th }}$ pandemic El Tor lineage has been

319 circulating in Asian and Middle Eastern countries for a long time (70), it is possible that, at least

320 within our dataset, the Mozambique isolates are the closest relative to this specific Pakistan

321 strain.

322

323 Confirmation of an African connection for the Yemen outbreak

324 The Yemen cholera outbreak began in October 2016 with eleven confirmed cases

325 (http://www.emro.who.int/pandemic-epidemic-diseases/cholera/cholera-cases-in-yemen.html). 
326 By January 2017, there were already over 10,000 cholera cases with 99 associated deaths

327 (http://www.emro.who.int/pandemic-epidemic-diseases/cholera/weekly-update-cholera-cases-in-

328 yemen-15-jan-2017.html). By the end of that year, there were over 900,000 cholera cases

329 (http://www.emro.who.int/pandemic-epidemic-diseases/cholera/outbreak-update-cholera-in-

330 yemen-19-december-2017.html). The outbreak continues on today as the largest cholera outbreak

331 in modern history. As isolates from this outbreak were only recently made available (9), they

332 were not part of the initial dataset for the cgMLST scheme development. These isolates were

333 added and analyzed on PubMLST after the scheme had been established. This set of isolates

334 therefore serves as an independent test of the universality and applicability of the cgMLST

335 scheme. To determine the potential origin of the Yemen outbreak and its phylogenetic

336 relationships with existing $V$. cholerae strains, the Yemen isolates were compared with other $7^{\text {th }}$

337 pandemic El Tor isolates from Asian and African countries (Table S3). All allele designations

338 and cgST assignments were done automatically on PubMLST. MST was built using these

339 isolates and all connections with seven and fewer allelic differences are represented as solid lines

340 (Fig. 6). Isolates connected by solid lines therefore belong in the same outbreak cluster as

341 defined by the outbreak threshold of seven allelic differences. Isolates from Yemen, Kenya, and

342 Haiti all cluster with the central Indian isolates with seven or fewer allelic differences; however,

343 the closest relatives to the Yemen isolates are those from Kenya with four or fewer allelic

344 differences (Fig. 6). The Indian isolates are the next closest connection but there is no direct

345 linkage between these and the Yemen isolate. This pattern is consistent with the work of Weill

346 and colleagues (9), where they suggested that the Yemen outbreak strains may have come from

347 East Africa which itself came from South Asia based on SNP-base phylogenetic analysis and

348 Bayesian evolutionary analysis. 
Unlike the limited samples available from African cholera outbreaks, the Haiti and

350 Yemen outbreaks are significant cases for epidemiological investigations because $V$. cholerae

351 has been heavily sampled from these countries as well as surrounding regions. Two major

352 limitations in genomic epidemiology have been the lack of a universal classification scheme and

353 a comprehensive database; however, this is no longer the case in the genomic era as sequencing

354 technology is becoming increasingly more accessible (8). A genomic approach, as shown here, is

355 able to produce accurate predictions of potential origins of outbreaks and provides us with

356 sufficient resolution to accurately track the spread of the disease. Therefore, genomic analysis

357 should be the first step in any epidemiological study as not only will it help guide subsequent

358 analyses and investigations, but consistently sequencing new genomes will also help expand and

359 refine the current global $V$. cholerae genome database.

361 Increased resolution for the history of cholera in Mozambique: comparing cgMLST to

362 MLVA

363 The $7^{\text {th }}$ pandemic reached Africa in 1970 and cholera appeared in Mozambique at roughly the

364 same time (57). Since its introduction, cholera has been endemic in that country and has

365 continued to cause multiple outbreaks (23). A popular tool for outbreak investigation is MLVA

$366(32,38)$, which was recently used to study $V$. cholerae strains collected in Mozambique over

367 multiple years (23). MLVA is a subspecies typing method similar to MLST in concept; however,

368 it utilizes VNTRs instead of using gene sequences. As VNTR mutates at a faster rate than

369 conserved genes, it has been shown that MLVA provides greater resolution than MLST for some

370 species $(32,33)$. To establish a direct comparison between our cgMLST scheme and this MLVA

371 scheme, we examined the MSTs created by both methods focusing only on shared isolates (Fig. 
372 7A and 7B). The MLVA identified 26 profiles forming two clonal complexes (CCs) and four

373 singletons (Fig. 7A) (23). A similar population structure is seen with the cgMLST analysis (Fig.

374 7B), including the four singletons identified in the MLVA. The central node in the cgMLST

375 MST consists mostly of isolates with MLVA profile ' $8,4,6,18,21$ ' similar to the central node in

376 the MLVA MST (23). The two CCs identified in the MLVA MST are also identified in the

377 cgMLST MST with the smaller CC2 being at least four allelic differences away from the larger

378 CC1.

Although there are a few MLVA types that were grouped into a single cgST, such as

380 cgST1 and cgST114, indicating that cgMLST was unable to resolve the differences in these

381 MLVA types, there are many MLVA types such as profile '2,4,6,18,21', profile '7,4,6,16,22',

382 profile ' $9,4,6,18,24$ ', and profile ' $8,4,6,18,22$ ' that were split into multiple cgSTs. Overall there

383 are $48 \mathrm{cgST}$ as opposed to only 26 MLVA types, showing that cgMLST provides better

384 resolution overall than MLVA. The cgMLST analysis overlaid with isolation dates shows that

385 the Mozambique $V$. cholerae strains are highly clonal and strains from the same cgST can cause

386 outbreaks over multiple years (e.g., cgST114 and cgST94) (Fig. 7C), which corroborates the

387 claim made in the initial MLVA study that the same MLVA type can be seen over multiple years

388 (23). In addition to increased resolution, cgMLST also produces more reliable and reproducible

389 results than MLVA, as it eliminates errors associated with the detection of VNTR regions using

390 PCR or sequencing-based methods. For the same reason that MLST is less affected by

391 convergent evolution compared to MLVA (35), cgMLST is also less affected by convergent

392 evolution. 


\section{Standardizing the genotypes responsible for the Haiti 2010 cholera outbreak: comparing}

\section{5 cgMLST and SNP-based analyses}

396 One of the largest cholera outbreaks in modern history occurred in Haiti following the

397 devastating earthquake in $2010(8,71)$. Prior to this outbreak, there were no documented cholera

398 cases in Haiti $(14,18)$. Since the initial introduction, $V$. cholerae now remains endemic in Haiti

399 and is responsible for thousands of cholera cases annually (71). Multiple studies have strongly

400 suggested that the Haitian strains were in fact imported from Nepal (by the UN Nepalese troops)

401 and the outbreak occurred as a result of both inappropriate sanitary practice and the lack of

402 screening of the UN troops upon their arrival in Haiti $(8,15,16,71)$.

A SNP-based approach was used to study the evolutionary dynamics of $V$. cholerae in

404 Haiti (14). This technique relies on the identification of SNPs in draft or closed genomes. The

405 primary benefit of this method is that assembly and annotation are not required. It is also capable

406 of resolving closely related strains using whole genome data. However, SNP-based methods are

407 highly influenced by recombination events $(43,44)$ and quality filter parameters chosen $(72)$.

To establish a direct comparison between the cgMLST scheme and SNP-based analysis,

we focused on MSTs of only the Haitian outbreak isolates (Fig. 8). All Haitian isolates are

410 closely related according to the cgMLST scheme, sharing at most four allelic differences with

411 each other (Fig. 8A). The Haitian and Nepalese isolates, therefore, also belong to the same

412 sublineage (SL6) which is consistent with the fact that these isolates belong to the same MLST

413 ST (either ST1 or ST69 based on the 2016 or 2013 MLST scheme, respectively (20, 21)) (Table

$414 \mathrm{~S} 1)$. The overall population structure is similar between the two methods where we have SNP

415 ST1 as the center of the MST with ST2 and ST3 extending from that likely ancestral genotype

416 (Fig. 8). SNP ST1, ST2, and ST3 can be split into 11, 2, and 3 different cgSTs, respectively (Fig. 
$4178 \mathrm{~A})$. There is only one case, that of cgST66, where it contains isolates from both SNP ST1 and

418 ST3. Overall, cgMLST was able to differentiate $39 \%$ of the isolates while the SNP-based

419 analysis differentiated 35\%, showing comparable level of resolution. As expected, both the

420 cgMLST and the SNP-based analyses showed that the Haiti outbreak is highly clonal where most

421 isolates belong to the same cgST or SNP ST (14). However, one important advantage of

422 cgMLST over SNP-based analysis is that the former can be easily standardized because it relies

423 on a predefined set of core genes. Based on these standardized genes, a systematic nomenclature

424 system can be established. This makes cgMLST more suitable than the SNP-based method as a

425 universally applicable classification system for epidemiological studies and research worldwide.

427 Environmental isolates differ from clinical strains by their diversity and their associations

428 with specific geographical locations

429 To look at the geographic signal of $V$. cholerae, we eliminated all clinical isolates and those that

430 belong to the PG lineage $(18,19)$. This is because the geographic signal of clinical strains can be

431 skewed, as pathogenic strains can travel long distances in a short period of time through

432 association with human hosts. The geographical analysis was therefore performed only with

433 environmental isolates.

434 Along with all the publicly available environmental strains that are not part of the PG

435 lineage, there are a total of 195 isolates spanning 9 countries. After grouping the isolates at the

436 sublineage level (i.e., each cluster have at most 133 allelic differences), it could be noted that all

437 isolates from the same sublineage also shared a country of origin, with the exception of strains

438 692-79 and 857 (Fig. 9), which are from the USA and Bangladesh, respectively. Phylogenetic

439 analysis shows these isolates to be closely related to strain A215, a clinical isolate from the USA 
440 (Fig. 4). All three strains contain the toxR gene, a toxin transcriptional regulator common in

441 pathogenic $V$. cholerae (73), as well as genes encoding for the Mannose-sensitive hemagglutinin

442 pilus, the RTX toxin, and hemolysin $(h l y A)$, all of which are putative virulence factors for this

443 species. In addition, strains A215 and 857 also harbor the zona occludens toxin gene. Similar

444 toxin gene contents among these three isolates and close phylogenetic relationships suggest that

445 strains 692-79 and 857 may also be potentially pathogenic and capable of surviving inside a

446 human host. This provides evidence that although clinical isolates can spread across the world

447 rapidly and closely related isolates can be from very different parts of the world, environmental

448 isolates from the same geographic origin share an affinity among each other at least at the

449 sublineage level. It is important to note that our current dataset contains a relatively small

450 number of environmental isolates that are not part of the PG lineage. Therefore, this distinct

451 distribution pattern based on geographic origin may be a result of currently insufficient sampling

452 of environmental $V$. cholerae worldwide. With large-scale environmental sampling, it will be

453 possible to determine with greater accuracy the evolutionary rate and distribution pattern of $V$.

454 cholerae in the environment using cgMLST. In addition, this method will become an invaluable

455 tool in dealing with these large datasets, as it provides an efficient and standardized method of

456 classification.

\section{Conclusion}

459 With an extensive collection of over 1,200 V. cholerae isolates, we developed a cgMLST scheme

460 based on 2,443 core genes. We established a sublineage-level definition based on 133 allelic

461 differences as part of our standardized classification scheme. It was determined by comparisons

462 with previous MLST schemes that the cgMLST sublineage classification can be used as a proxy 
463 for traditional MLST. Additionally, the universality and applicability of the scheme have been

464 tested by looking at various cholera outbreak cases. We determined an outbreak threshold based

465 on seven allelic differences that groups isolates from the same outbreak together with strains

466 from the potential source of introduction. This threshold creates clusters that are consistent with

467 known epidemiological data when applied to the Haiti and Yemen cholera outbreaks, two of the

468 best-documented cholera outbreaks in modern history. Also, we were able to confirm the South

469 Asian origin of modern cholera outbreaks. Furthermore, although current sampling is limited, a

470 geographic signal at the sublineage level not seen in clinical strains could be identified among

471 environmental isolates that are not part of the PG lineage $(18,19)$. Lastly, this scheme is fully

472 implemented on PubMLST (https://pubmlst.org/vcholerae/) for public access. All newly

473 available genomes uploaded to PubMLST will be annotated automatically and a cgST

474 designation will be assigned to isolates with less than 100 missing loci. Relevant epidemiological

475 data and the variety of analytical and visualization tools are all integrated on PubMLST,

476 allowing for a quick analysis of any newly sequenced genome in a global context. This scheme

477 will be an important tool for future large-scale epidemiological and biogeographical research.

479 MATERIALS AND METHODS

480 Dataset description

481 On November $6^{\text {th }} 2018,1,172 \mathrm{~V}$. cholerae genomes consisting of 800 draft and complete

482 genomes and 372 sequence read archives (SRAs), available from both publicly available

483 database and private collection, were selected as our dataset. One hundred sixteen SRAs from a

484 recent study on the Yemen cholera outbreak (9) were subsequently added as an independent

485 evaluation of the cgMLST scheme (Table S3). The 488 SRAs were assembled using skesa (74) 
or the CLC Genomics Workbench 7 (QIAGEN) using default parameters. This total dataset of 1,288 included twenty-six genomes with less than $90 \%$ of the core genes, which were identified using USearch (75) based on RAST (76) annotations. These 26 genomes were removed from

489 subsequent analyses resulting in a final dataset of 1,262 genomes collected from 52 countries and 490 spanning 82 years from 1937 to 2018 (Table S1). These include a historical collection from the $4916^{\text {th }}$ cholera pandemic, clinical isolates from outbreaks in various countries (e.g., Bangladesh, 492 India, Haiti, Yemen, the Democratic Republic of Congo, and Russia), and environmental isolates 493 from different parts of the world (e.g., Bangladesh, Haiti, USA, Mexico, Brazil, etc.).

\section{Gene identification and allele assignments}

496 Instead of using the full dataset of 1,288 genomes, we selected a subset of high-quality genomes

497 because core gene identification is highly dependent on the initial dataset and the inclusion of

498 poorly assembled and/or sequenced data will reduce the number of core genes identified (49).

499 Firstly, 800 already assembled draft or complete genomes were selected for core gene

500 identification. Low-quality assemblies were then eliminated by removing genomes with less than

$50140 \times$ coverage and/or N50 values less than $40 \mathrm{~kb}$. From a previously established cgMLST scheme

502 for L. monocytogenes, $40 \times$ coverage and $20 \mathrm{~kb} \mathrm{N50}$ value were used as cutoff thresholds, as

503 genomes that do not meet these criteria resulted in a low proportion of loci being called (49). The

$50440 \times$ coverage cutoff was adopted for this study; however, because the average $V$. cholerae

505 genome size $(\sim 4 \mathrm{Mb})$ is larger than the average L. monocytogenes genome $(\sim 3 \mathrm{Mb}), 40 \mathrm{~kb}$ was

506 instead selected as the N50 cutoff. The use of these cutoffs resulted in the removal of 82

507 genomes. 
The remaining 718 genomes were annotated using RAST (76) and USearch (75), and a tentative set of core genes that were on average present in $99 \%$ of the genomes were selected. An

510 additional 13 genomes were removed, as they lacked more than $90 \%$ for the core genes, leaving

511 us with a dataset of 705 high-quality genomes (Table S4). However, an additional 26 genomes

512 were subsequently removed for the core gene analysis as it has been previously suggested that

513 they form a highly divergent lineage within the $V$. cholerae (77-79), ensuring that the dataset

514 used for core gene identification consists only of unambiguously $V$. cholerae isolates (also as

515 verified by average nucleotide identity (80) and digital DNA-DNA hybridization (81) between

516 genomes (77-79)). Completeness and potential contamination of all remaining 679 genomes

517 were also independently evaluated by checkM, which estimates these values based on the

518 presence and number of copies of a set of pre-defined single copy marker genes (82) (Table S5).

519 All genomes were, according to the criteria established by checkM, nearly complete ( $\geq 97 \%)$ with

520 medium to low levels of contamination $(<7 \%)(82)$.

521 Each orthologous gene was compared against the $V$. cholerae N16961 reference genome

522 using BLASTN (83) to determine gene function. Any gene family with no homolog in N16961

523 or classified as pseudogenes on the NCBI GenBank database were removed, meaning N16961

524 was $100 \%$ complete for the cgMLST scheme. Any gene that was present in more than one copy

525 in any of the initial 679 genomes was also removed, as they were considered paralogous. Thus,

526 in this context, core genes are defined as being present in at least $90 \%$ of the 679 high-quality

527 assembled genomes in a single copy. By choosing a relaxed cutoff of $90 \%$ completeness, we

528 accounted for missing genes due to sequencing, annotation, or assembly errors while ensuring

529 there is sufficient resolution to differentiate between closely related strains, with at least 2,199

530 loci remaining for classification purposes. The final cgMLST scheme utilizes a set of 2,443 
531 single-copy core gene loci, which is $2,425,296 \mathrm{bp}$ in size and covering approximately $61 \%$ of the

532 genome. The list of core genes is available on PubMLST (https://pubmlst.org/vcholerae/).

534 all 1,262 isolates (Table S1). Allele calls were made only for complete coding sequences with a

535 minimum of $70 \%$ similarity and $70 \%$ length coverage at the nucleotide level, as previously

536 described (49). Default settings were used for all other parameters.

\section{Core genome sequence type (cgST) assignment}

539 cgST, which was defined as a unique combination of alleles of all loci included in the scheme,

540 was assigned for all isolates, excluding those from the Yemen outbreak study (8), with an in-

541 house script, as previously described (85). Briefly, missing loci were replaced with the most

542 common allele when assigning cgSTs, allowing for a conservative estimate of diversity (85). The

543116 isolates from the Yemen cholera outbreak study (9) were annotated automatically by

544 uploading them to PubMLST. PubMLST treated missing alleles as 'N'. cgSTs were assigned to

545 each allele profile, treating ' $\mathrm{N}$ ' as a regular allele designation. However, different from typical

546 allele designations, 'N's can represent any allelic sequence; therefore, some isolates may contain

547 multiple cgST designations, all of which are possibly true cgSTs. For isolates with more than one

548 cgST suggested by PubMLST, postprocessing was done using an in-house script to identify the

549 most likely cgST, which was determined by assuming missing loci contained the most common

550 allele (Table S2). It is expected that as genome sequencing becomes more reliable, higher quality

551 genomes will be available and any missing data can be updated as needed. 


\section{MLST scheme and sequence type (ST) assignments}

554 Two MLST schemes developed for $V$. cholerae were mapped to this cgMLST scheme. The first

555 MLST scheme developed in 2013 by Octavia and colleagues (20) was used to study the global

556 population structure of non-O1/non-O139 V. cholerae and is currently hosted on PubMLST. All

557 isolates uploaded to PubMLST were automatically annotated with this scheme. Any missing data

558 in this scheme was ignored and no ST designation was assigned. The second MLST scheme

559 developed in 2016 by Kirchberger and colleagues (21) was used to study the population structure

560 of environmental $V$. cholerae in a region on the US East Coast. The second MLST scheme is not

561 currently hosted on PubMLST, but because the housekeeping genes in this scheme are also

562 found in the cgMLST scheme, a similar in-house script used in cgST assignments was used to

563 assign ST designations. Therefore, all isolates in this study were assigned three designations

564 when possible - two ST designations based on the two previously established MLST schemes

$565(20,21)$ and one cgST designation based on the cgMLST scheme from this study.

\section{Outbreak and sublineage clustering thresholds}

568 A clustering threshold was defined as the maximum number of allelic differences found within a

569 cluster. All clusters were produced based on the single-linkage clustering method, which meant

570 an isolate belonged to a cluster if it linked with any isolate within that cluster. Two metrics were

571 used as general guidelines for determining clustering thresholds. The first metric used was the

572 Dunn Index (DI), which measured clustering efficiencies (60). Briefly, the DI was highest for a

573 network (i.e., the network has the best clustering efficiency) when the intra-cluster distances

574 were minimized, and the inter-cluster distances were maximized. Since isolate distances were

575 measured based on allelic differences, a high DI resulted in clusters where isolates were more 
576 closely related to those found within the same cluster than those found in a different cluster. The

577 DI was calculated using the R packages 'clvalid' and 'boot' with 100 bootstrap replicates for

578 each threshold and graphed using the R package 'ggplot2' (86-89).

579 The second metric used was the Adjusted Rand Index (ARI), which measured the level of

580 similarity between two networks when clustering the same set of isolates by measuring the

581 amount of agreements (i.e., the number of pairs that were grouped either as being in the same

582 cluster or different cluster in both networks) and disagreements (i.e., the number of pairs that

583 were grouped together in one network but grouped separately in another) (62). The values ranged

584 from -1 (i.e., two networks are exactly opposite) to 1 (i.e., two networks are identical). ARI was

585 used to determine the level of similarity between various clustering thresholds and the MLST

586 schemes. ARI was calculated using the R package 'clues' and graphed using 'ggplot2' (87, 89,

$58790)$

589 Minimum spanning tree (MST)

590 All MSTs, unless otherwise specified, were constructed using GrapeTree MSTv2 (91). Loci with

591 missing data were included in the profile as "-". GrapeTree provided a novel algorithm that

592 accounted for missing data when constructing an MST, an important feature since missing data is

593 common in whole and core genome-based analyses. GrapeTree is currently integrated within

594 PubMLST, which allows for quick visualization of the dataset with any provenance data.

\section{Phylogenetic analysis}

597 Parsnp v1.2 (92) was used to reconstruct the phylogenetic tree using $V$. cholerae N16961 as the

598 reference genome. The -x flag was used to enable filtering of SNPs in recombinogenic regions as 
599 identified by PhiPack (93). Default settings were used for all other parameters. The phylogenetic

600 tree included 1,146 genomes (all genomes except for the 116 isolates from the recent Yemen

601 cholera outbreak study (9)). Since all isolates sequenced for the latter study belonged to the $7^{\text {th }}$

602 pandemic El Tor lineage, it would have had limited impact on the overall structure of the tree.

603 The phylogeny was visualized and annotated using iTOL (94).

\section{Biogeographical analysis of environmental isolates}

606 All environmental isolates that were not part of the PG lineage $(18,19)$ were first clustered based

607 on the sublineage threshold using the python package 'networkX' (95). Missing alleles were

608 replaced with the most common allelic designation when calculating pairwise differences to

609 establish a more conservative estimate of diversity. The network was then visualized using

610 Cytoscape (96).

612 Data availability

613 All previously sequenced $V$. cholerae genomes and the additional 65 genomes sequenced in this

614 study are available on the NCBI GenBank database. Table S6 lists all the accession numbers for

615 all the genomes used in this study. In addition, all genome sequences, allelic profiles, cgST

616 designations, ST designations, and relevant epidemiological data are publicly available on

617 PubMLST (https://pubmlst.org/vcholerae/). 


\section{ACKNOWLEDGMENTS}

620 KYHL, FDO, and YFB conceived the experiments. KYHL, FDO, and MTI performed all data

621 collection and analyses. FDO and TN performed genome sequencing of Bangladesh isolates. MA

622 and CLT provided isolates used in this study.

623 We thank Dr. Keith Jolley (University of Oxford) for providing valuable feedback

624 regarding the development of the cgMLST scheme, as well as the implementation of this scheme

625 on PubMLST. We also thank Monica Im (Centers for Disease Control and Prevention) for

626 assistance with obtaining whole genome sequences.

627 This work was supported by the Natural Sciences and Engineering Research Council

628 (NSERC) of Canada (to YFB); the Integrated Microbial Biodiversity program of the Canadian

629 Institute for Advanced Research (to YFB); federal appropriations to the Centers for Disease

630 Control and Prevention through the Advanced Molecular Detection Initiative (to CLT); and

631 graduate student scholarships from Alberta Innovates - Technology Futures (to KYHL, FDO,

632 MTI, and TN), NSERC (to KYHL and TN), the University of Alberta Faculty of Graduate

633 Studies and Research (Queen Elizabeth II Graduate Scholarship to KYHL and TN), and the

634 Bank of Montréal Financial Group (to FDO). MA of icddr,b acknowledges the governments of

635 Bangladesh, Canada, Sweden, and the United Kingdom for providing core/unrestricted support.

636 The funders had no role in study design, data collection and interpretation, or the decision to

637 submit the work for publication. The findings and conclusions in this report are those of the

638 authors and do not necessarily represent the official position of the Centers for Disease Control

639 and Prevention. 


\section{REFERENCES}

641 1. Jahan S. 2016. Cholera - epidemiology, prevention and control, p. 145-157. In Makun, HA 642 (ed.), Significance, Prevention and Control of Food Related Diseases. Croatia: InTech. 643 InTechOpen, Rijeka, Croatia.

2. Momba M, Azab El-Liethy M. 2017. Vibrio cholerae and Cholera biotypes, p. online. In

3. Clemens JD, Nair GB, Ahmed T, Qadri F, Holmgren J. 2017. Cholera. Lancet 390:1539-

4. Kaper JB, Morris JG, Levine MM. 1995. Cholera. Clin Microbiol Rev 8:48-86.

5. Islam MT, Alam M, Boucher Y. 2017. Emergence, ecology and dispersal of the pandemic

6. Choi SY, Rashed SM, Hasan NA, Alam M, Islam T, Sadique A, Johura F-T, Eppinger M,

7. Dalsgaard A, Skov MN, Serichantalergs O, Echeverria P, Meza R, Taylor DN. 1997.

8. Orata FD, Keim PS, Boucher Y. 2014. The 2010 Cholera Outbreak in Haiti: How Science Solved a Controversy. PLoS Pathog 10:e1003967.

9. Weill F-X, Domman D, Njamkepo E, Almesbahi AA, Naji M, Nasher SS, Rakesh A, Assiri AM, Sharma NC, Kariuki S, Pourshafie MR, Rauzier J, Abubakar A, Carter JY, Wamala JF, Seguin C, Bouchier C, Malliavin T, Bakhshi B, Abulmaali HH., Kumar D, Njoroge SM, Malik MR, Kiiru J, Luquero FJ, Azman AS, Ramamurthy T, Thomson NR, Quilici M-L. 2018. Genomic insights into the 2016-2017 cholera epidemic in Yemen. Nature 565:230233.

674 13. Sack DA, Sack RB, Chaignat C-L. 2006. Getting Serious about Cholera. N Engl J Med 
355:649-651.

14. Katz LSS, Petkau A, Beaulaurier J, Tyler S, Antonova ESS, Turnsek MAA, Guo Y, Wang S, Paxinos EEE, Orata F, Gladney LMM, Stroika S, Folster JPP, Rowe L, Freeman MMM, Knox N, Frace M, Boncy J, Graham M, Hammer BKK, Boucher Y, Bashir A, Hanage WPP, Domselaar GV Van, Tarr L, Van Domselaar G, Tarr CLL, Domselaar GV Van. 2013. Evolutionary Dynamics of Vibrio cholerae O1 following a Single-Source Introduction to Haiti. MBio 4:e00398-13.

15. Frerichs RR. 2016. Deadly river: Cholera and cover-up in post-earthquake Haiti. Cornell University Press, Ithaca.

16. Frerichs RR, Keim PS, Barrais R, Piarroux R. 2012. Nepalese origin of cholera epidemic in Haiti. Clin Microbiol Infect 18:E158-E163.

17. Hendriksen RS, Price LB, Schupp JM, Gillece JD, Kaas RS, Engelthaler DM, Bortolaia V, Pearson T, Waters AE, Prasad Upadhyay B, Devi Shrestha S, Adhikari S, Shakya G, Keim PS, Aarestrup FM. 2011. Population Genetics of Vibrio cholerae from Nepal in 2010: Evidence on the Origin of the Haitian Outbreak. MBio 2:e0157-11.

18. Boucher Y. 2016. Sustained Local Diversity of Vibrio cholerae O1 Biotypes in a Previously Cholera-Free Country. MBio 7:e0570-16.

19. Chun J, Grim CJ, Hasan NA, Lee JH, Choi SY, Haley BJ, Taviani E, Jeon Y-S, Kim DW, Lee J-H, Brettin TS, Bruce DC, Challacombe JF, Detter JC, Han CS, Munk AC, Chertkov O, Meincke L, Saunders E, Walters RA, Huq A, Nair GB, Colwell RR. 2009. Comparative genomics reveals mechanism for short-term and long-term clonal transitions in pandemic Vibrio cholerae. Proc Natl Acad Sci 106:15442-15447.

20. Octavia S, Salim A, Kurniawan J, Lam C, Leung Q, Ahsan S, Reeves PR, Nair GB, Lan R. 2013. Population Structure and Evolution of Non-O1/Non-O139 Vibrio cholerae by Multilocus Sequence Typing. PLoS One 8:e65342.

21. Kirchberger PC, Orata FD, Barlow EJ, Kauffman KM, Case RJ, Polz MF, Boucher Y. 2016. A small number of phylogenetically distinct clonal complexes dominate a coastal Vibrio cholerae population. Appl Environ Microbiol 82:5576-5586.

22. Bwire G, Sack DA, Almeida M, Li S, Voeglein JB, Debes AK, Kagirita A, Buyinza AW, Orach CG, Stine OC. 2018. Molecular characterization of Vibrio cholerae responsible for cholera epidemics in Uganda by PCR, MLVA and WGS. PLoS Negl Trop Dis 12:e006492.

711 24. Safa A, Nair GB, Kong RYC. 2010. Evolution of new variants of Vibrio cholerae O1. 
Trends Microbiol 18:46-54.

713
25. Boucher Y, Orata FD, Alam M. 2015. The out-of-the-delta hypothesis: Dense human populations in low-lying river deltas served as agents for the evolution of a deadly pathogen. Front Microbiol 6:1120.

26. Mandal S, Mandal MD, Pal NK. 2011. Cholera: A great global concern. Asian Pac J Trop Med 4:573-580.

27. Maiden MCJ, Bygraves JA, Feil E, Morelli G, Russell JE, Urwin R, Zhang Q, Zhou J, Zurth K, Caugant DA, Feavers IM, Achtman M, Spratt BG. 1998. Multilocus sequence typing: A portable approach to the identification of clones within populations of pathogenic microorganisms. Proc Natl Acad Sci USA 95:3140-3145.

28. Horwood P, Collins D, Jonduo M, Rosewell A, Dutta S, Dagina R, Ropa B, Siba P, Greenhill A. 2011. Clonal Origins of Vibrio cholerae O1 El Tor Strains, Papua New Guinea, 2009-2011. Emerg Infect Dis 17:2063.

29. Luo Y, Ye J, Jin D, Ding G, Zhang Z, Mei L, Octavia S, Lan R. 2013. Molecular analysis of non-O1/non-O139 Vibrio cholerae isolated from hospitalised patients in China. BMC Microbiol 13:52.

30. Maiden MCJ, Van Rensburg MJJ, Bray JE, Earle SG, Ford SA, Jolley KA, McCarthy ND. 2013. MLST revisited: The gene-by-gene approach to bacterial genomics. Nat Rev Microbiol 11:728-736.

31. Gonzalez-Escalona N, Martinez-Urtaza J, Romero J, Espejo T R, Jaykus L-A, DePaola A. 2008. Determination of Molecular Phylogenetics of Vibrio parahaemolyticus Strains by Multilocus Sequence Typing. J Bacteriol 190:2831-2840.

32. Lam C, Octavia S, Reeves PR, Lan R. 2012. Multi-locus variable number tandem repeat analysis of 7th pandemic Vibrio cholerae. BMC Microbiol 12:82.

33. Chenal-Francisque V, Passet V, Brisse S, Cantinelli T, Diancourt L, Pourcel C, Lecuit M, Leclercq A, Tran-Hykes C, Bracq-Dieye H. 2013. Optimized Multilocus Variable-Number Tandem-Repeat Analysis Assay and Its Complementarity with Pulsed-Field Gel Electrophoresis and Multilocus Sequence Typing for Listeria monocytogenes Clone Identification and Surveillance. J Clin Microbiol 51:1868-1880.

34. Vogler AJ, Birdsell DN, Lee J, Vaissaire J, Doujet CL, Lapalus M, Wagner DM, Keim P. 2011. Phylogeography of Francisella tularensis ssp. holarctica in France. Lett Appl Microbiol 52:177-180.

35. Struelens MJ, Brisse S. 2013. From molecular to genomic epidemiology: Transforming surveillance and control of infectious diseases. Eurosurveillance 18:20386.

36. Sabat AJ, Budimir A, Nashev D, Sá-Leão R, van Dijl JM, Laurent F, Grundmann H, Friedrich AW, on behalf of the ESCMID Study Group. 2013. Overview of molecular 
typing methods for outbreak detection and epidemiological surveillance. Eurosurveillance 18:20380.

37. Klassen JL, Currie CR. 2012. Gene fragmentation in bacterial draft genomes: extent, consequences and mitigation. BMC Genomics 13:14.

38. Danin-Poleg Y, Cohen LA, Gancz H, Broza YY, Goldshmidt H, Malul E, Valinsky L, Lerner L, Broza M, Kashi Y. 2007. Vibrio cholerae Strain Typing and Phylogeny Study Based on Simple Sequence Repeats. J Clin Microbiol 45:736-746.

39. Wong VK, Baker S, Connor TR, Pickard D, Page AJ, Dave J, Murphy N, Holliman R, Sefton A, Millar M, Dyson ZA, Dougan G, Holt KE. 2016. An extended genotyping framework for Salmonella enterica serovar Typhi, the cause of human typhoid. Nat Commun 7:12827.

40. Leekitcharoenphon P, Nielsen EM, Kaas RS, Lund O, Aarestrup FM. 2014. Evaluation of Whole Genome Sequencing for Outbreak Detection of Salmonella enterica. PLoS One 9:e87991.

41. Chen C, Zhang W, Zheng H, Lan R, Wang H, Du P, Bai X, Ji S, Meng Q, Jin D, Liu K, Jing H, Ye C, Gao GF, Wang L, Gottschalk M, Xu J. 2013. Minimum core genome sequence typing of bacterial pathogens: A unified approach for clinical and public health microbiology. J Clin Microbiol 51:2582-2591.

42. Qin T, Zhang W, Liu W, Zhou H, Ren H, Shao Z, Lan R, Xu J. 2016. Population structure and minimum core genome typing of Legionella pneumophila. Sci Rep 6:21356.

43. Wang R, Yu D, Yue J, Kan B. 2016. Variations in SXT elements in epidemic Vibrio cholerae O1 El Tor strains in China. Sci Rep 6:22733.

44. Meibom KL, Blokesch M, Dolganov NA, Wu C-Y, Schoolnik GK. 2005. Chitin induces natural competence in Vibrio cholerae. Science 310:1824-1827.

45. Borgeaud S, Metzger LC, Scrignari T, Blokesch M. 2015. The type VI secretion system of Vibrio cholerae fosters horizontal gene transfer. Science 347:63-68.

46. Orata FD, Kirchberger PC, Méheust R, Barlow EJ, Tarr CL, Boucher Y. 2015. The Dynamics of Genetic Interactions between Vibrio metoecus and Vibrio cholerae, Two Close Relatives Co-Occurring in the Environment. Genome Biol Evol 7:2941-2954.

47. Boucher Y, Cordero OX, Takemura A, Hunt DE, Schliep K, Bapteste E, Lopez P, Tarr CL, Polz MF. 2011. Local Mobile Gene Pools Rapidly Cross Species Boundaries To Create Endemicity within Global Vibrio cholerae Populations. MBio 2.

48. Neumann B, Prior K, Bender JK, Harmsen D, Klare I, Fuchs S, Bethe A, Zühlke D, Göhler A, Schwarz S, Schaffer K, Riedel K, Wieler LH, Werner G. 2019. A Core Genome Multilocus Sequence Typing Scheme for Enterococcus faecalis. J Clin Microbiol 57:e01686-18. 
49. Moura A, Criscuolo A, Pouseele H, Maury MM, Leclercq A, Tarr C, Björkman JT, Dallman T, Reimer A, Enouf V, Larsonneur E, Carleton H, Bracq-Dieye H, Katz LS, Jones L, Touchon M, Tourdjman M, Walker M, Stroika S, Cantinelli T, Chenal-Francisque V, Kucerova Z, Rocha EPC, Nadon C, Grant K, Nielsen EM, Pot B, Gerner-Smidt P, Lecuit M, Brisse S. 2016. Whole genome-based population biology and epidemiological surveillance of Listeria monocytogenes. Nat Microbiol 2:1-10.

50. de Been M, Pinholt M, Top J, Bletz S, Mellmann A, van Schaik W, Brouwer E, Rogers M, Kraat Y, Bonten M, Corander J, Westh H, Harmsen D, Willems RJL. 2015. Core Genome Multilocus Sequence Typing Scheme for High-Resolution Typing of Enterococcus faecium. J Clin Microbiol 53:3788-3797.

51. Cody AJ, Bray JE, Jolley KA, McCarthy ND, Maiden MCJ. 2017. Core Genome Multilocus Sequence Typing Scheme for Stable, Comparative Analyses of Campylobacter jejuni and C. coli Human Disease Isolates. J Clin Microbiol 55:2086-2097.

52. Janowicz A, De Massis F, Ancora M, Camma C, Patavino C, Battisti A, Prior K, Harmsen D, Scholz H, Zilli K, Sacchini L, Di Giannatale E, Garofolo G. 2018. Core Genome Multilocus Sequence Typing and Single Nucleotide Polymorphism Analysis in the Epidemiology of Brucella melitensis Infections. J Clin Microbiol 56:e00517-18.

53. Bletz S, Janezic S, Harmsen D, Rupnik M, Mellmann A. 2018. Defining and Evaluating a Core Genome Multilocus Sequence Typing Scheme for Genome-Wide Typing of Clostridium difficile. J Clin Microbiol 56:e01987-17.

54. Jones RC, Harris LG, Morgan S, Ruddy MC, Perry M, Williams R, Humphrey T, Temple M, Davies AP. 2019. Phylogenetic Analysis of Mycobacterium tuberculosis Strains in Wales by Use of Core Genome Multilocus Sequence Typing To Analyze Whole-Genome Sequencing Data. J Clin Microbiol 57:e02025-18.

55. Sails AD, Swaminathan B, Fields PI. 2003. Clonal complexes of Campylobacter jejuni identified by multilocus sequence typing correlate with strain associations identified by multilocus enzyme electrophoresis. J Clin Microbiol 41:4058-4067.

56. Leavis HL, Bonten MJ, Willems RJ. 2006. Identification of high-risk enterococcal clonal complexes: global dispersion and antibiotic resistance. Curr Opin Microbiol 9:454-460.

57. Weill F-X, Domman D, Njamkepo E, Tarr C, Rauzier J, Fawal N, Keddy KH, Salje H, Moore S, Mukhopadhyay AK, Bercion R, Luquero FJ, Ngandjio A, Dosso M, Monakhova E, Garin B, Bouchier C, Pazzani C, Mutreja A, Grunow R, Sidikou F, Bonte L, Breurec S, Damian M, Njanpop-Lafourcade B-M, Sapriel G, Page A-L, Hamze M, Henkens M, Chowdhury G, Mengel M, Koeck J-L, Fournier J-M, Dougan G, Grimont PAD, Parkhill J, Holt KE, Piarroux R, Ramamurthy T, Quilici M-L, Thomson NR. 2017. Genomic history of the seventh pandemic of cholera in Africa. Science 358:785-789.

58. Reimer A, Domselaar G, Stroika S, Walker M, Kent H, Tarr C, Talkington D, Rowe L, Olsen-Rasmussen M, Frace M, Sammons S, Dahourou G, Boncy J, Smith A, Mabon P, Petkau A, Graham M, Gilmour M, Gerner-Smidt P. 2011. Comparative Genomics of Vibrio 
cholerae from Haiti, Asia, and Africa. Emerg Infect Dis 17:2113.

824

825

826

827

828

829

830

831

832

833

834

835

836

837

838

839

840

841

842

843

844

845

846

59. Salim A, Lan R, Reeves PR. 2005. Vibrio cholerae pathogenic clones. Emerg Infect Dis 11:1758-1760.

60. Dunn JC. 1974. Well-separated clusters and optimal fuzzy partitions. J Cybern 4:95-104.

61. Mutreja A, Kim DW, Thomson NR, Connor TR, Lee JH, Kariuki S, Croucher NJ, Choi SY, Harris SR, Lebens M, Niyogi SK, Kim EJ, Ramamurthy T, Chun J, Wood JLN, Clemens JD, Czerkinsky C, Nair GB, Holmgren J, Parkhill J, Dougan G. 2011. Evidence for several waves of global transmission in the seventh cholera pandemic. Nature 477:462-465.

62. Hubert L, Arabie P. 1985. Comparing partitions. J Classif 2:193-218.

63. Lucidarme J, Hill DMC, Bratcher HB, Gray SJ, du Plessis M, Tsang RSW, Vazquez JA, Taha M-K, Ceyhan M, Efron AM, Gorla MC, Findlow J, Jolley KA, Maiden MCJ, Borrow R. 2015. Genomic resolution of an aggressive, widespread, diverse and expanding meningococcal serogroup B, C and W lineage. J Infect 71:544-552.

64. Royer G, Fourreau F, Boulanger B, Mercier-Darty M, Ducellier D, Cizeau F, Potron A, Podglajen I, Mongardon N, Decousser J-W. 2019. Local outbreak of extended-spectrum $\beta$ lactamase SHV2a-producing Pseudomonas aeruginosa reveals the emergence of a new specific sub-lineage of the international ST235 high-risk clone. J Hosp Infect.

65. Eppinger M, Pearson T, Koenig SSK, Pearson O, Hicks N, Agrawal S, Sanjar F, Galens K, Daugherty S, Crabtree J, Hendriksen RS, Price LB, Upadhyay BP, Shakya G, Fraser CM, Ravel J, Keim PS. 2014. Genomic Epidemiology of the Haitian Cholera Outbreak: a Single Introduction Followed by Rapid, Extensive, and Continued Spread Characterized the Onset of the Epidemic. MBio 5:e01721-14.

66. World Health Organization. 2017. Weekly epidemiological record.

67. Cavailler P, Lucas M, Perroud V, McChesney M, Ampuero S, Guérin PJ, Legros D, Nierle T, Mahoudeau C, Lab B, Kahozi P, Deen JL, von Seidlein L, Wang XY, Puri M, Ali M, Clemens JD, Songane F, Baptista A, Ismael F, Barreto A, Chaignat CL. 2006. Feasibility of a mass vaccination campaign using a two-dose oral cholera vaccine in an urban choleraendemic setting in Mozambique. Vaccine 24:4890-4895.

68. Sardar T, Mukhopadhyay S, Bhowmick AR, Chattopadhyay J. 2013. An optimal cost effectiveness study on Zimbabwe cholera seasonal data from 2008-2011. PLoS One 8:e81231.

69. Hasan NA, Choi SY, Eppinger M, Clark PW, Chen A, Alam M, Haley BJ, Taviani E, Hine E, Su Q, others. 2012. Genomic diversity of 2010 Haitian cholera outbreak strains. Proc Natl Acad Sci 109:E2010--E2017.

70. Hu D, Liu B, Feng L, Ding P, Guo X, Wang M, Cao B, Reeves PR, Wang L. 2016. Origins of the current seventh cholera pandemic. Proc Natl Acad Sci 113:E7730-E7739. 
71. Guillaume Y, Ternier R, Vissieres K, Casseus A, Chery MJ, Ivers LC. 2018. Responding to cholera in Haiti: Implications for the national plan to eliminate cholera by 2022. J Infect Dis 218:S167-S170.

72. Pightling AW, Petronella N, Pagotto F. 2014. Choice of Reference Sequence and Assembler for Alignment of Listeria monocytogenes Short-Read Sequence Data Greatly Influences Rates of Error in SNP Analyses. PLoS One 9:e104579.

73. Childers BM, Klose KE. 2007. Regulation of virulence in Vibrio cholerae: The ToxR regulon. Future Microbiol 2:335-344.

74. Souvorov A, Agarwala R, Lipman DJ. 2018. SKESA: strategic k-mer extension for scrupulous assemblies. Genome Biol 19:153.

75. Edgar RC. 2010. Search and clustering orders of magnitude faster than BLAST.

76. Aziz RK, Bartels D, Best AA, DeJongh M, Disz T, Edwards RA, Formsma K, Gerdes S, Glass EM, Kubal M, Meyer F, Olsen GJ, Olson R, Osterman AL, Overbeek RA, McNeil LK, Paarmann D, Paczian T, Parrello B, Pusch GD, Reich C, Stevens R, Vassieva O, Vonstein V, Wilke A, Zagnitko O, Formsma K, Kubal M, Vonstein V, Stevens R, McNeil LK, Edwards RA, Pusch GD, Reich C, Glass EM, Olsen GJ, Paczian T, Overbeek RA, Meyer F, Vassieva O, DeJongh M, Osterman AL, Disz T, Best AA, Gerdes S, Parrello B, Bartels D, Olson R, Paarmann D. 2008. The RAST server: rapid annotations using subsystems technology. BMC Genomics 9:75.

77. Islam MT, Liang K, Im MS, Winkjer J, Busby S, Tarr CL, Boucher Y. 2018. Draft Genome Sequences of Nine Vibrio sp. Isolates from across the United States Closely Related to Vibrio cholerae. Microbiol Resour Announc 7:e00965-18.

78. Liang K, Islam MT, Hussain N, Winkjer NS, Im MS, Rowe LA, Tarr CL, Boucher Y. 2019. Draft Genome Sequences of Eight Vibrio sp. Clinical Isolates from across the United States That Form a Basal Sister Clade to Vibrio cholerae. Microbiol Resour Announc 8:e0147318 .

79. Liang K, Orata FD, Winkjer NS, Rowe LA, Tarr CL, Boucher Y. 2017. Complete Genome Sequence of Vibrio sp. Strain 2521-89, a Close Relative of Vibrio cholerae Isolated from Lake Water in New Mexico, USA. Genome Announc 5:e00905-17.

80. Goris J, Konstantinidis KT, Klappenbach JA, Coenye T, Vandamme P, Tiedje JM. 2007. DNA-DNA hybridization values and their relationship to whole-genome sequence similarities. Int J Syst Evol Microbiol 57:81-91.

81. Meier-Kolthoff JP, Auch AF, Klenk HP, Göker M. 2013. Genome sequence-based species delimitation with confidence intervals and improved distance functions. BMC Bioinformatics 14:60.

82. Parks DH, Imelfort M, Skennerton CT, Hugenholtz P, Tyson GW. 2015. CheckM : 
assessing the quality of microbial genomes recovered from isolates, single cells, and metagenomes. Genome Res 25:1043-1055.

83. Altschul SF, Gish W, Miller W, Myers EW, Lipman DJ. 1990. Basic local alignment search tool. J Mol Biol 215:403-410.

84. Jolley KA, Maiden MCJ. 2010. BIGSdb: Scalable analysis of bacterial genome variation at

85. Garg P, Aydanian A, Smith D, Morris JGJG, Nair GB, Stine OC, Antonia A, Smith D, Morris JGJG, Nair GB, Stine OC. 2003. Molecular Epidemiology of O139 Vibrio cholerae: Mutation, Lateral Gene Transfer, and Founder Flush. Emerg Infect Dis 9:810-814.

86. Brock G, Pihur V, Datta S, Datta S. 2008. clValid: An R Package for Cluster Validation. J Stat Softw 25:1-22.

87. R Core Team. 2017. R: A Language and Environment for Statistical Computing. Vienna, Austria.

88. Canty A, Ripley BD, others. 2017. boot: Bootstrap R (S-Plus) Functions. R Packag version 1.

89. Wickham H. 2009. ggplot2: Elegant Graphics for Data Analysis. Springer-Verlag New

90. Chang F, Qiu W, Zamar RH, Lazarus R, Wang X. 2010. clues: An R Package for Nonparametric Clustering Based on Local Shrinking. J Stat Softw 33:1-16.

91. Zhou Z, Alikhan N, Sergeant MJ, Luhmann N, Vaz C, Francisco AP, Carriço JA, Achtman M. 2018. GrapeTree: visualization of core genomic relationships among 100,000 bacterial pathogens. Genome Res 28:1395-1404.

92. Treangen TJ, Ondov BD, Koren S, Phillippy AM. 2014. The harvest suite for rapid coregenome alignment and visualization of thousands of intraspecific microbial genomes. Genome Biol 15:524.

93. Bruen TC, Philippe H, Bryant D. 2006. A simple and robust statistical test for detecting the presence of recombination. Genetics 172:2665-2681.

94. Letunic I, Bork P. 2007. Interactive Tree Of Life (iTOL): An online tool for phylogenetic

95. Hagberg A, Swart P, S Chult D. 2008. Exploring network structure, dynamics, and function using NetworkX, p. 11-16. In Varoquaux G, Vaught T, Millman J (ed), Proceedings of the 7th Python in Science Conference (SciPy2008).

96. Shannon P, Markiel A, Ozier O, Baliga NS, Wang JT, Ramage D, Amin N, Schwikowski B, Ideker T. 2003. Cytoscape: A Software Environment for Integrated Models of 
bioRxiv preprint doi: https://doi.org/10.1101/2020.01.27.919118; this version posted February 21, 2020. The copyright holder for this preprint (which was not certified by peer review) is the author/funder, who has granted bioRxiv a license to display the preprint in perpetuity. It is made available under aCC-BY 4.0 International license.

$930 \quad$ Biomolecular Interaction Networks. Genome Res 13:2498-2504.

931 


\section{FIGURE LEGENDS}

933 Fig. 1. Pairwise allelic differences for all isolates used in this study. Both plots show the

934 frequency of allelic mismatches in pairwise comparisons. A) Pairwise comparisons of up to

935 2,443 allelic differences are shown. Major peaks are shaded. B) Comparisons with up to 500

936 allelic differences are shown. Pairwise comparisons of only clinical isolates are shown in red.

937 Vertical lines indicate the outbreak threshold (red) and sublineage threshold (blue).

939 Fig. 2. Plot showing the Dunn Index for clustering thresholds ranging from 1 to 1,000 allelic

940 differences. Each clustering threshold is bootstrapped 100 times. The median, plotted with the

941 light blue shade, indicates the $25^{\text {th }}$ to $75^{\text {th }}$ percentile range. Red and blue vertical lines indicate

942 the outbreak and sublineage thresholds, respectively. The dotted lines represent other clustering

943 thresholds used in the Adjusted Rand Index calculations (Fig. 3B and Fig. S3).

945 Fig. 3. Evaluation of network similarities between the cgMLST sublineage threshold and MLST

946 ST. A) Networks of all sublineages identified using only $V$. cholerae isolates from Bangladesh ( $n$

$947=255$ ). Each cluster represents a sublineage and includes isolates with less than or equal to 133

948 allelic differences with each other. Each node represents a cgST and is colored by sequence type

949 based on the 2016 MLST scheme (21). Size of the nodes are proportional to the number of

950 isolates. The length of the connecting lines within a cluster is proportional to the number of

951 allelic differences. B) Adjusted Rand Index for individual pairwise comparisons between

952 predefined clustering thresholds (Fig. 2) and the 2016 MLST scheme (21). The sublineage

953 clustering threshold (i.e., 133 allelic differences) and outbreak threshold (i.e., 7 allelic

954 differences) are indicated in blue and red bars, respectively. 
955 Fig. 4. Phylogenetic tree of 1,146 V. cholerae isolates (excluding the 116 isolates from the

956 Yemen cholera outbreak) reconstructed using Parsnp v1.2 (92). All group inside the PG lineage

957 ( $7^{\text {th }}$ pandemic El Tor, El Tor progenitor, El Tor sister, Classical, and Classical sister) are

958 collapsed. Outer rings represents clustering by sequence type based on the 2016 MLST scheme

959 by Kirchberger and colleagues (21), whereas the inner ring represents clustering based on the

960 sublineage threshold (i.e., 133 allelic differences). Branches of clinical strains are colored in red.

961 The phylogenetic tree is rooted with a basal lineage to $V$. cholerae (collapsed) $(77,78)$.

962

963 Fig. 5. Minimum spanning trees isolated when the outbreak threshold was applied to the

964 complete dataset of 1,262 isolates. A) All isolates that clustered together with the Haiti (dark

965 blue) and Yemen (light blue) isolates based on the clustering threshold of seven allelic

966 differences. B) All isolates that clustered with the Mozambique isolates (dark blue) based on the

967 clustering threshold of seven allelic differences. Additional Mozambique isolates that are not

968 part of the same outbreak cluster are also shown. Three isolates, two from Zimbabwe (green) and

969 one from the USA (orange), are connected as they share seven or fewer allelic differences with

970 the Mozambique isolates. In both panels, the size of the nodes is proportional to the number of

971 isolates. Length of the lines is proportional to the number of allelic differences and all

972 connections have less than or equal to seven allelic differences.

973

974 Fig. 6. cgMLST MST of all Yemen isolates and representative $7^{\text {th }}$ pandemic El Tor strains. All

975 isolates connected by dotted lines share eight or more allelic differences (not drawn to scale). All

976 isolates connected with solid lines share seven or fewer allelic differences (i.e., they belong to

977 the same outbreak cluster; drawn to scale). Each node represents a cgST that is colored by year 
978 of collection. The outbreak clusters are shaded by country.

979

980 Fig. 7. Comparison between cgMLST and MLVA with a focus on the Mozambique isolates. A)

981 Population structure of pandemic $V$. cholerae in Mozambique based on MLVA profiles by

982 Garrine and colleagues (23). MST of the Mozambique isolates based on the cgMLST scheme

983 colored based on B) MLVA profiles and C) year of isolation. All isolates in B and C connected

984 with lines share seven or fewer allelic differences. For all panels, the size of the nodes is

985 proportional to the number of isolates. The length of the lines is proportional to the number of

986 allelic differences.

987

988 Fig. 8. Comparison between cgMLST and SNP-based analysis with a focus on the Haiti outbreak

989 and related isolates. A) MST of isolates from the 2010 cholera outbreak in Haiti. All lines

990 indicate connections of four or fewer allelic differences. Each node represents a cgST which is

991 colored by year of isolation. Background shading represent ST designations based on 45 high-

992 quality SNPs by Katz and colleagues (14). Note that cgST66 contains a mix of color as it

993 contains both ST1 and ST3. Any isolate from countries other than Haiti is indicated. The length

994 of the lines is proportional to the number of allelic differences. B) MST constructed from whole

995 genome SNP data (14). The length of the lines indicates the number of nucleotide substitutions.

996 Size of the nodes is proportional to the number of isolates.

997

998 Fig. 9. Sublineage clusters of non-clinical environmental isolates that are not part of the PG

999 lineage. Clusters are constructed using networkX (95) and visualized with Cytoscape (96).

1000 Missing loci were assumed to contain the most common allele when calculating allelic 
bioRxiv preprint doi: https://doi.org/10.1101/2020.01.27.919118; this version posted February 21, 2020. The copyright holder for this preprint

(which was not certified by peer review) is the author/funder, who has granted bioRxiv a license to display the preprint in perpetuity. It is made available under aCC-BY 4.0 International license.

1001 differences. Isolates are connected only if they share 133 allelic differences or fewer with each

1002 other. Each node represents an isolate and is colored by the country of origin. 

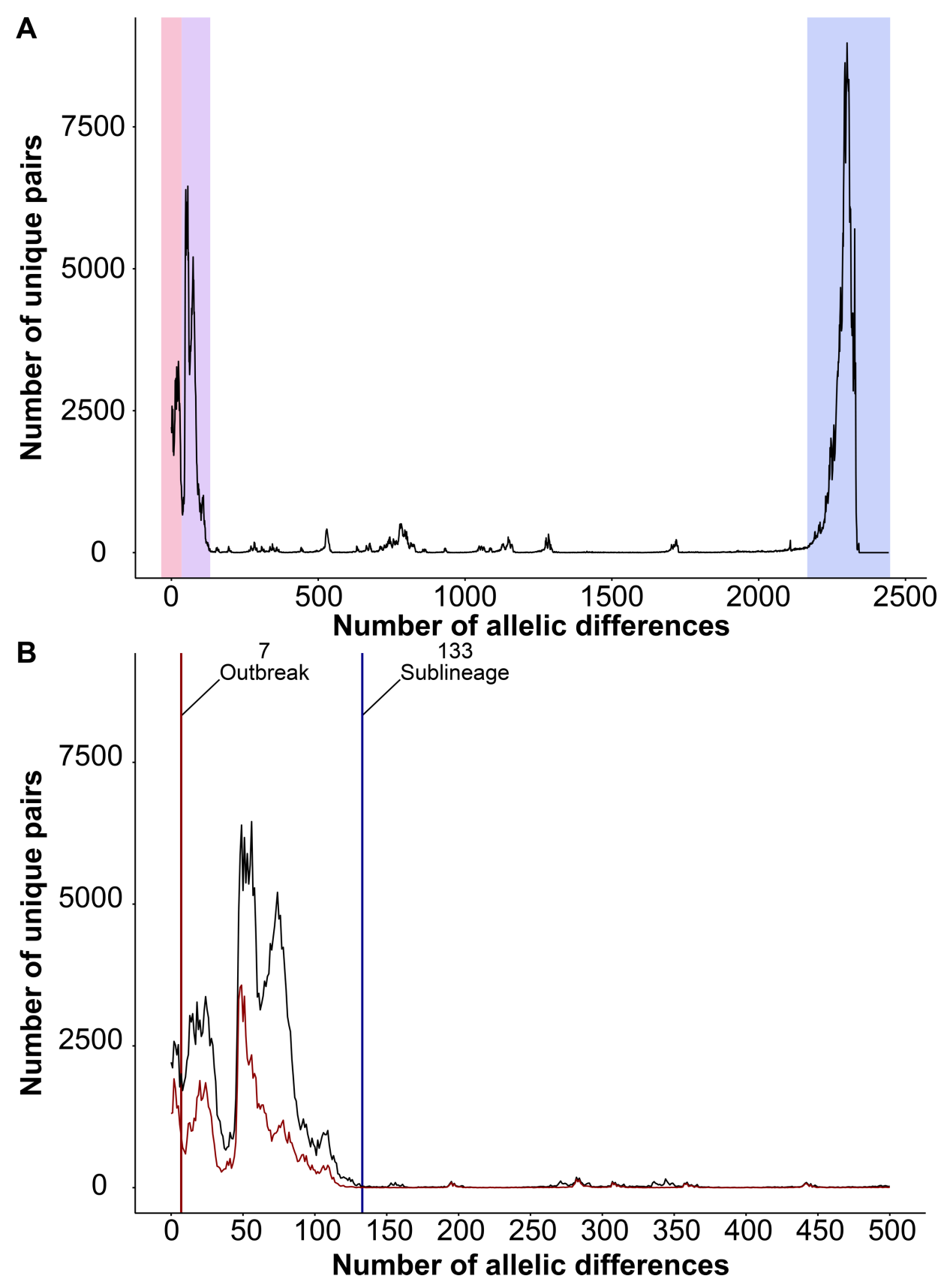

1005 Fig. 1. Pairwise allelic differences for all isolates used in this study. Both plots show the

1006 frequency of allelic mismatches in pairwise comparisons. A) Pairwise comparisons of up to

1007 2,443 allelic differences are shown. Major peaks are shaded. B) Comparisons with up to 500

1008 allelic differences are shown. Pairwise comparisons of only clinical isolates are shown in red.

1009 Vertical lines indicate the outbreak threshold (red) and sublineage threshold (blue). 


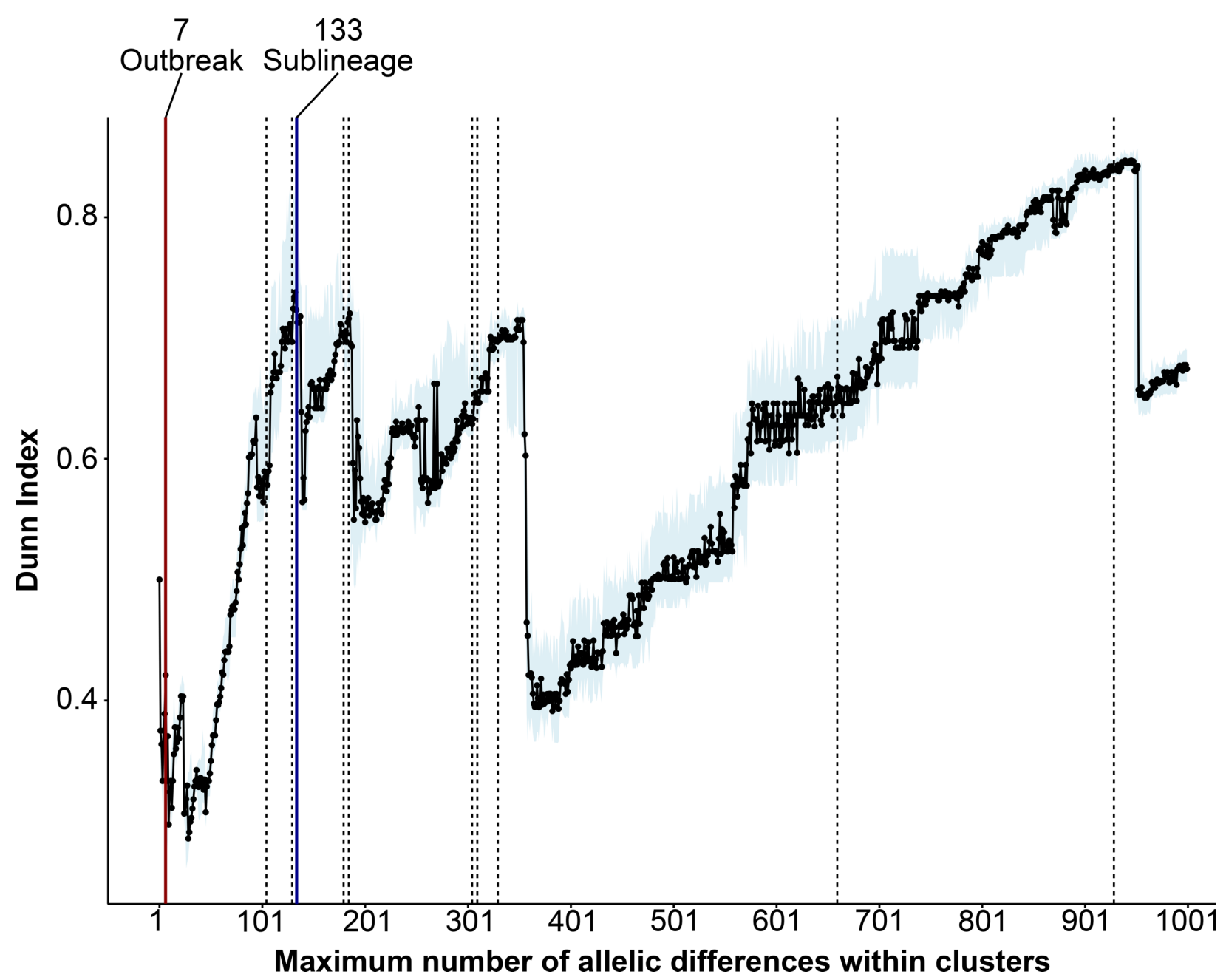

1011 Fig. 2. Plot showing the Dunn Index for clustering thresholds ranging from 1 to 1,000 allelic

1012 differences. Each clustering threshold is bootstrapped 100 times. The median, plotted with the

1013 light blue shade, indicates the $25^{\text {th }}$ to $75^{\text {th }}$ percentile range. Red and blue vertical lines indicate

1014 the outbreak and sublineage thresholds, respectively. The dotted lines represent other clustering

1015 thresholds used in the Adjusted Rand Index calculations (Fig. 3B and Fig. S3). 
A

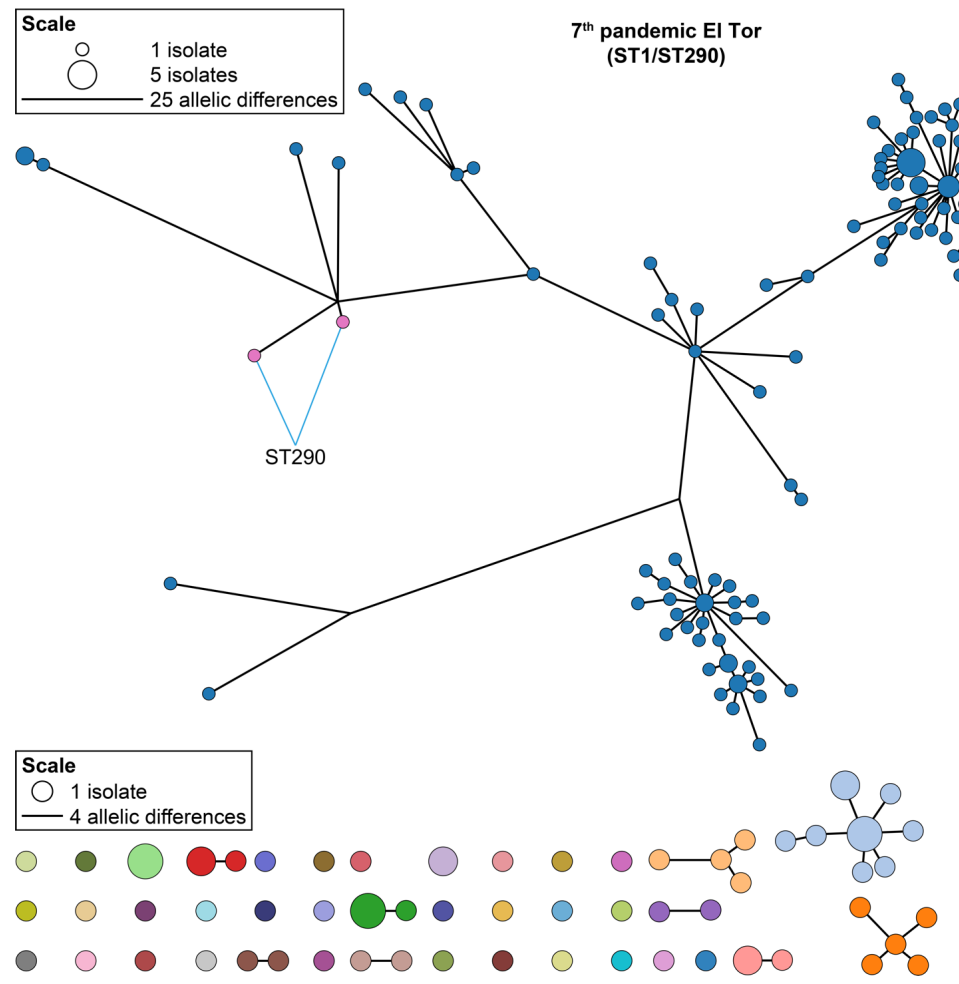

Sequence type [number of isolates]

(Kirchberger et al., 2016)

$\begin{array}{ll}\square 1[183] & \square 82[1] \\ \square 275[11] & \square 163[1]\end{array}$

$165[5]$

$223[4]$

164 [3]

$\square 11[2]$

$\square 176[3]$

$261[3]$

$250[2]$

$260[2]$

$284[2]$

$290[2]$

$13[1]$

$\square 146$ [1]

$\square 147$ [1]

$\square$
$\square 286$ [1]

286 [1]

$\square 54$ [1]

$\square 78$ [1]

$\square 177$ [1]
$\square 184$ [1]

$\square$
$\square$
$\square$

201 [1]

$\square$
$\square 202[1]$

$\square 210$ [1]

$\square 217$ [1]

$\square 224$ [1]

$\square 237$ [1]

$\square 21$ [1]
$\square 26$ [1]

$\square 26$ [1]
$\square 263$ [1]

266 [1]

$\square 267$ [1]

268 [1]
$\square 274[1]$

274 [1]

276 [1]

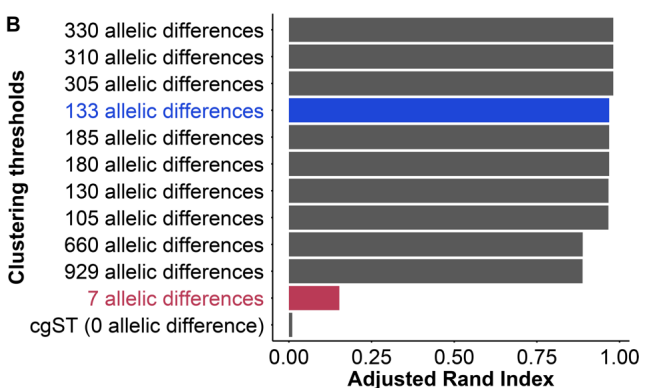

1017 Fig. 3. Evaluation of network similarities between the cgMLST sublineage threshold and MLST

1018 ST. A) Networks of all sublineages identified using only $V$. cholerae isolates from Bangladesh $(n$

$1019=255)$. Each cluster represents a sublineage and includes isolates with less than or equal to 133

1020 allelic differences with each other. Each node represents a cgST and is colored by sequence type

1021 based on the 2016 MLST scheme (21). Size of the nodes are proportional to the number of

1022 isolates. The length of the connecting lines within a cluster is proportional to the number of

1023 allelic differences. B) Adjusted Rand Index for individual pairwise comparisons between

1024 predefined clustering thresholds (Fig. 2) and the 2016 MLST scheme (21). The sublineage

1025 clustering threshold (i.e., 133 allelic differences) and outbreak threshold (i.e., 7 allelic

1026 differences) are indicated in blue and red bars, respectively. 


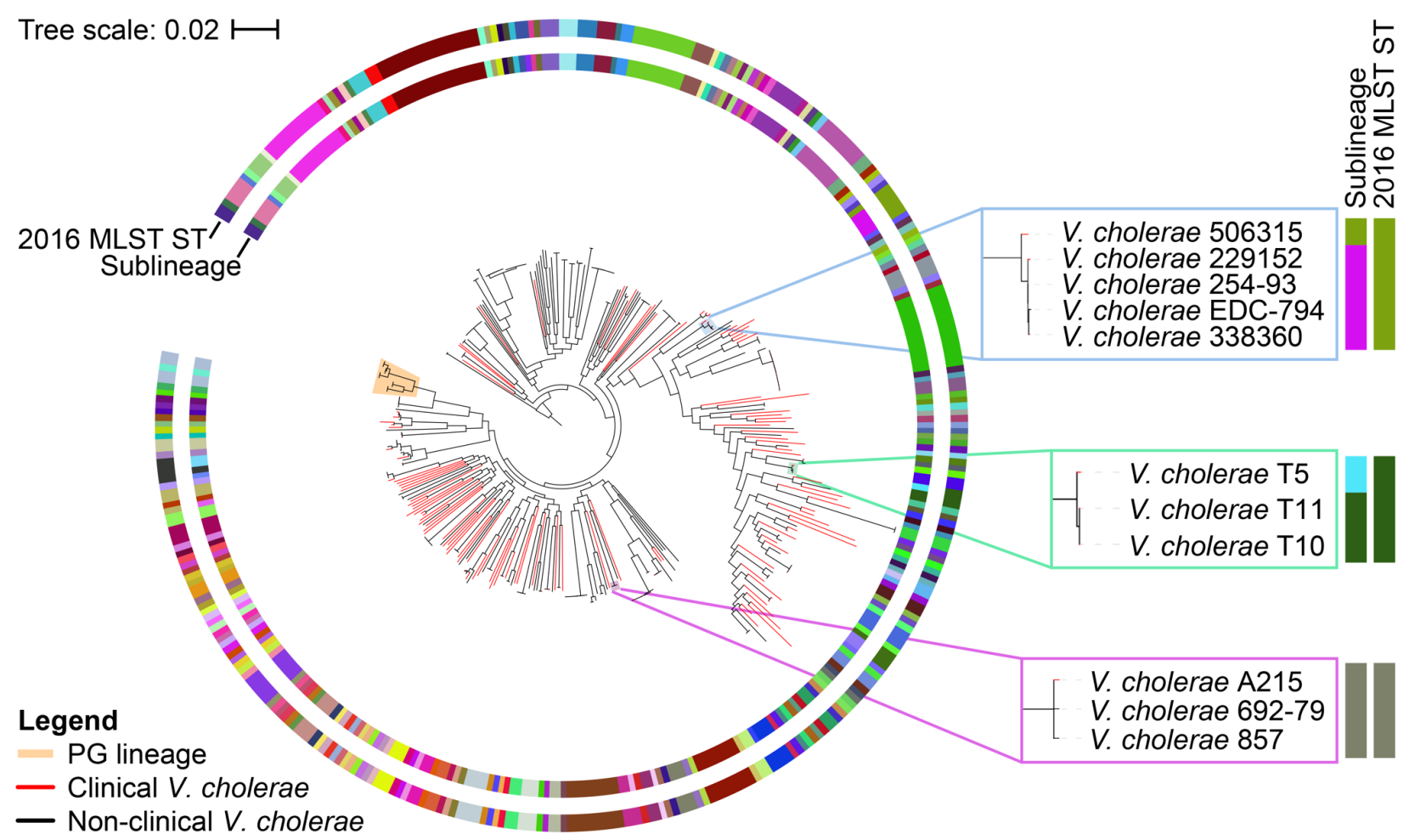

1028 Fig. 4. Phylogenetic tree of 1,146 V. cholerae isolates (excluding the 116 isolates from the

1029 Yemen cholera outbreak) reconstructed using Parsnp v1.2 (92). All group inside the PG lineage

$1030 \quad 7^{\text {th }}$ pandemic El Tor, El Tor progenitor, El Tor sister, Classical, and Classical sister) are

1031 collapsed. Outer rings represents clustering by sequence type based on the 2016 MLST scheme

1032 by Kirchberger and colleagues (21), whereas the inner ring represents clustering based on the

1033 sublineage threshold (i.e., 133 allelic differences). Branches of clinical strains are colored in red.

1034 The phylogenetic tree is rooted with a basal lineage to $V$. cholerae (collapsed) $(77,78)$. 
A

1036

1037

1038

1039

1040

1041

1042

1043

1044

1045

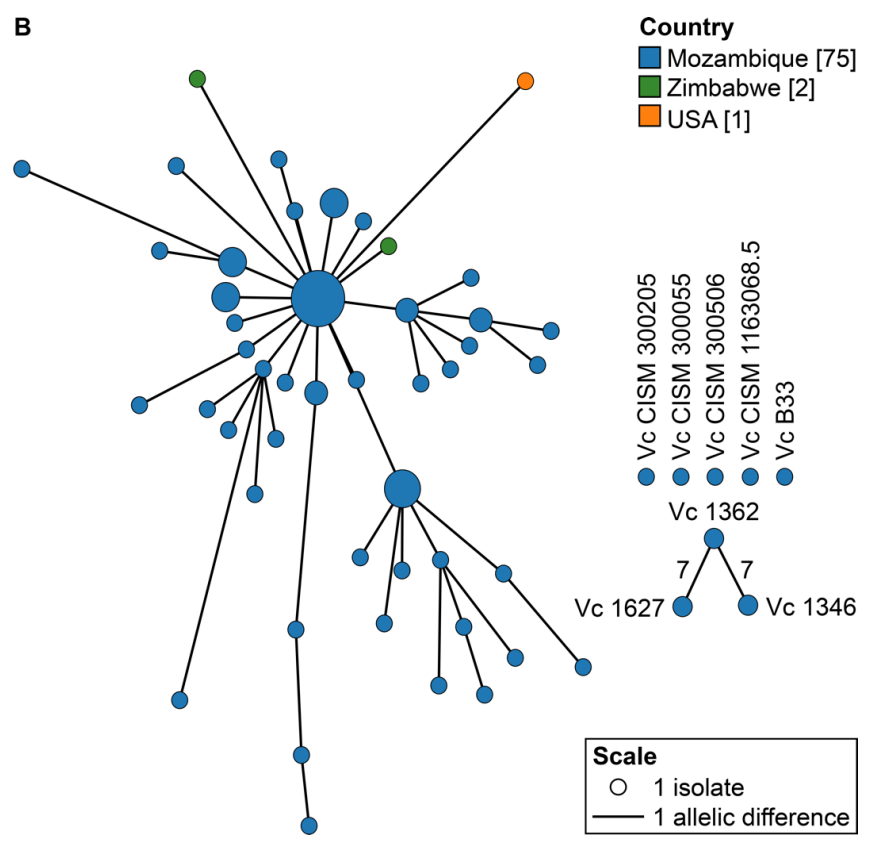

Fig. 5. Minimum spanning trees isolated when the outbreak threshold was applied to the complete dataset of 1,262 isolates. A) All isolates that clustered together with the Haiti (dark blue) and Yemen (light blue) isolates based on the clustering threshold of seven allelic differences. B) All isolates that clustered with the Mozambique isolates (dark blue) based on the clustering threshold of seven allelic differences. Additional Mozambique isolates that are not part of the same outbreak cluster are also shown. Three isolates, two from Zimbabwe (green) and one from the USA (orange), are connected as they share seven or fewer allelic differences with the Mozambique isolates. In both panels, the size of the nodes is proportional to the number of isolates. Length of the lines is proportional to the number of allelic differences and all connections have less than or equal to seven allelic differences. 


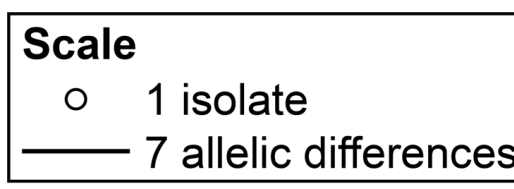

Country of origin

$\square$ Yemen

Kenya

India

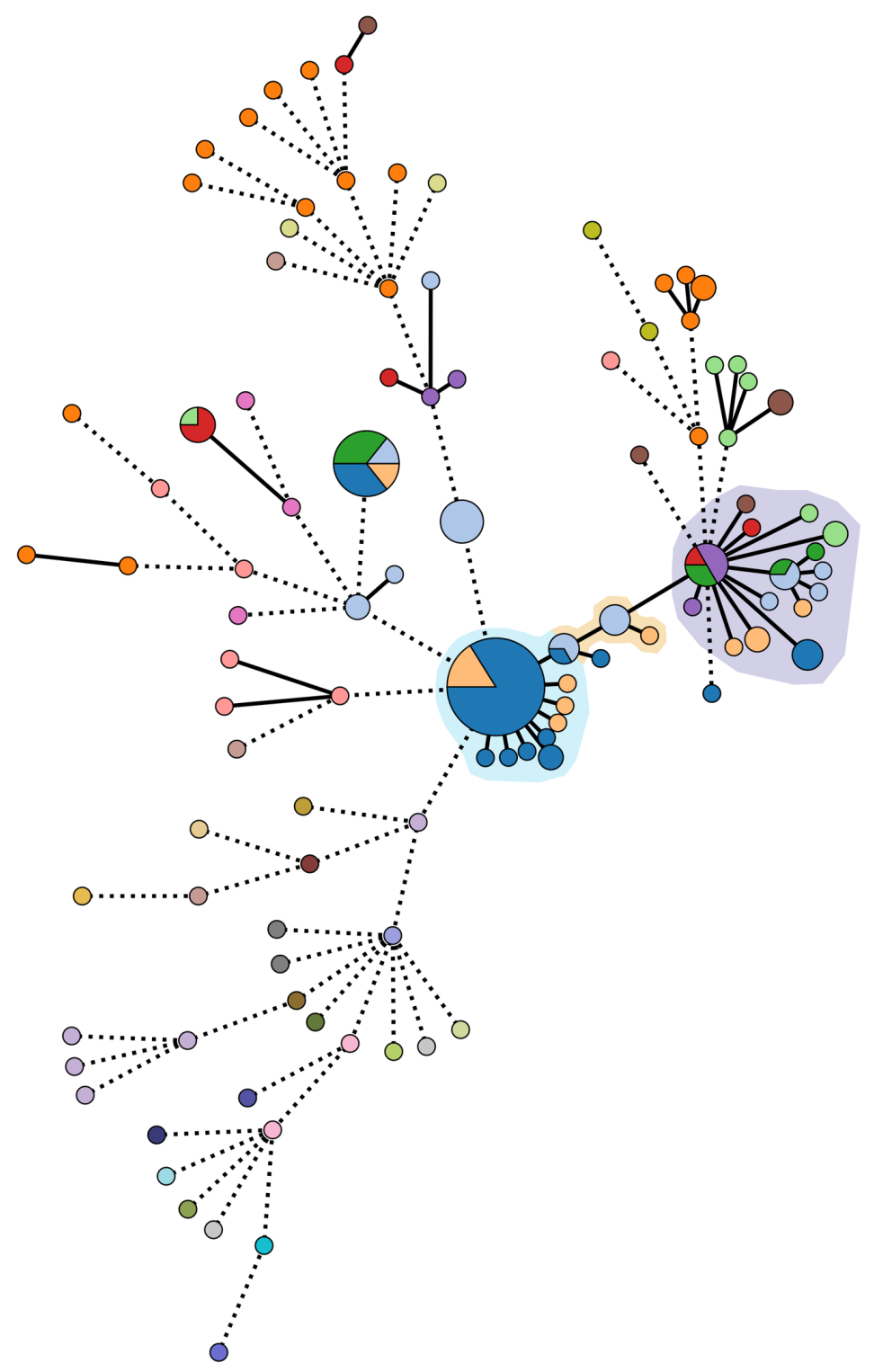

Year

$\square 2017$ [43]

$\square 2015$ [22]

$\square 2007$ [18]

2016 [15]

2014 [9]

$\square 2010$ [8]

2012 [7]

$\square 2005$ [6]

2013 [6]

$\square 1991$ [5]

$\square 2011$ [5]

2004 [3]

2009 [3]

$\square 1977$ [2]

1979 [2]

1985 [2]

2001 [2]

2006 [2]

$\square 1937$ [1]

1938 [1]

1954 [1]

1957 [1]

1966 [1]

1971 [1]

1973 [1]

1974 [1]

$\square 1975$ [1]

1980 [1]

1989 [1]

1992 [1]

1995 [1]

2002 [1]

2003 [1]

1047 Fig. 6. cgMLST MST of all Yemen isolates and representative $7^{\text {th }}$ pandemic El Tor strains. All

1048 isolates connected by dotted lines share eight or more allelic differences (not drawn to scale). All

1049 isolates connected with solid lines share seven or fewer allelic differences (i.e., they belong to

1050 the same outbreak cluster; drawn to scale). Each node represents a cgST that is colored by year

1051 of collection. The outbreak clusters are shaded by country. 
A Clonal complex 1

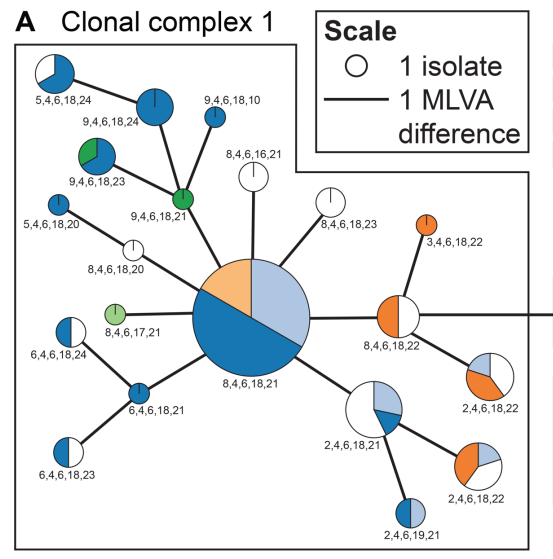

c

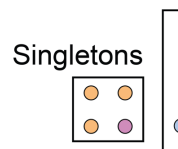

Clonal complex 1
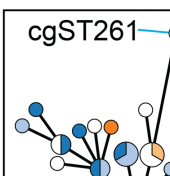

Year

$\square 2002 \square 2003$

$\square 2005 \square 2008$

$2009 \square 2010$

$2012 \square$ Unknown

Clonal complex 2
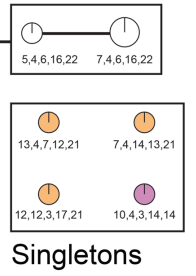

Year

$\square 2003$ [22]

$\square 2002$ [10]

$\square 2010$ [5]

$\square 2008$ [7]

2009 [2]

2005 [1]

$2012[1]$

$\square$ Unknown
B

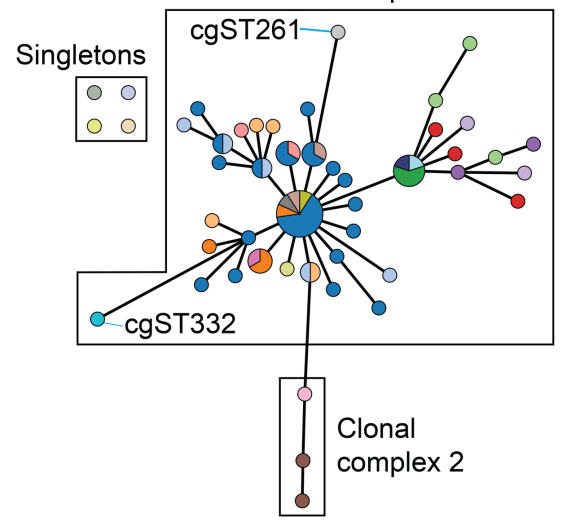

Scale

O 1 isolate

-1 allelic difference
MLVA

$8,4,6,18,21$ [26]

$2,4,6,18,21$ [5]

$2,4,6,18,22[4]$

$\square 8,4,6,18,22[4]$

$5,4,6,18,24[3]$

$9,4,6,18,23[3]$

$9,4,6,18,24$ [3]

$[2,4,6,19,21[2]$

$6,4,6,18,23[2]$

$6,4,6,18,24$ [2]

$7,4,6,16,22[2]$

$8,4,6,19,21[2]$

$\square 3,4,6,18,22$ [1]

$\square 5,4,6,16,22[1]$

$\square 6,4,6,18,21[1]$

$\square 8,4,6,16,21[1]$

$\square 8,4,6,17,21[1]$

$\square 8,4,6,18,20$ [1]

$\square 8,4,6,18,23[1]$

$\square 9,4,6,18,10$ [1]

$9,4,6,18,21[1]$

$\square 13,4,7,12,21$ [1]

$\square 7,4,14,13,21$ [1]

$\square 12,12,3,17,21$ [1]

$\square 10,4,3,14,14$ [1]

1053 Fig. 7. Comparison between cgMLST and MLVA with a focus on the Mozambique isolates. A)

1054 Population structure of pandemic $V$. cholerae in Mozambique based on MLVA profiles by

1055 Garrine and colleagues (23). MST of the Mozambique isolates based on the cgMLST scheme

1056 colored based on B) MLVA profiles and C) year of isolation. All isolates in B and C connected

1057 with lines share seven or fewer allelic differences. For all panels, the size of the nodes is

1058 proportional to the number of isolates. The length of the lines is proportional to the number of

1059 allelic differences. 
A Year
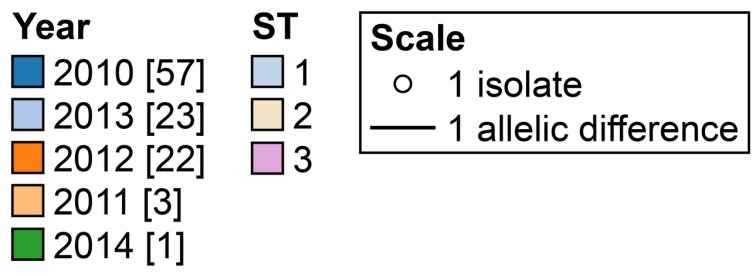

1 allelic difference
B

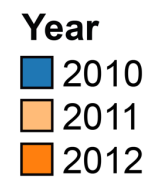

\section{Scale}

1 isolate

1 SNP difference

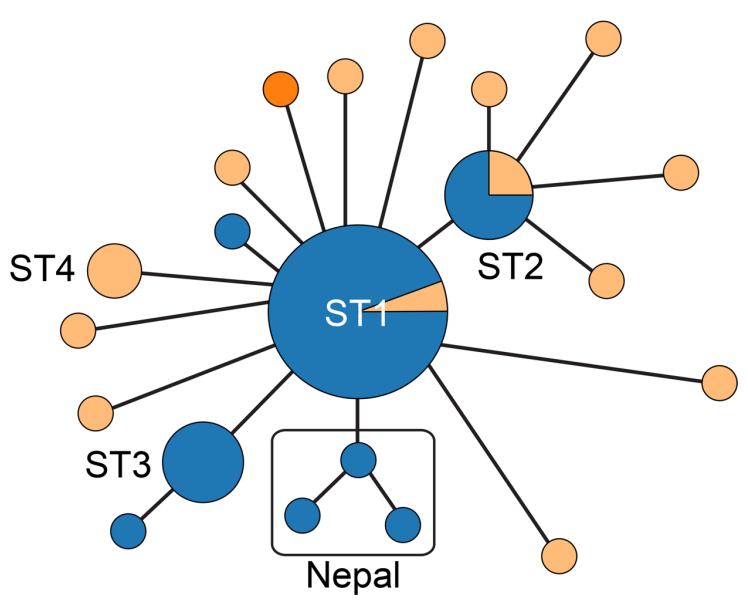

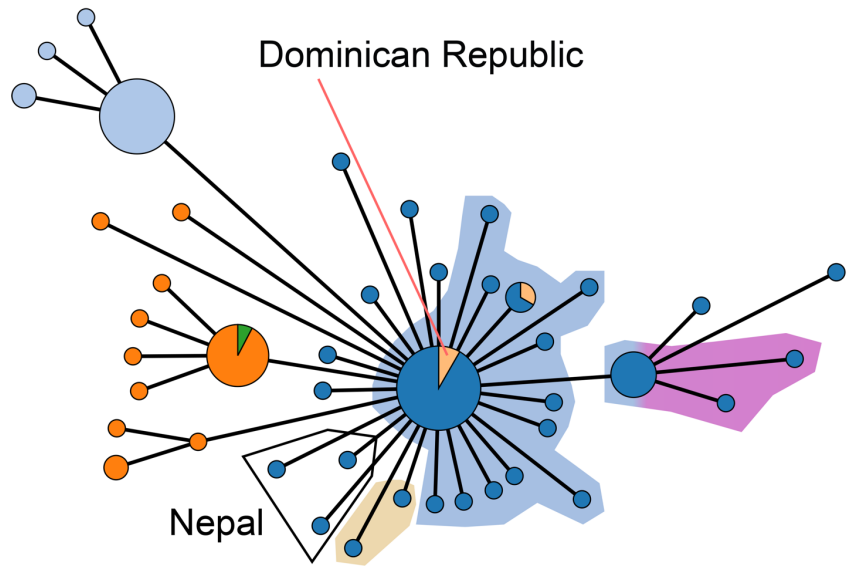

1062 and related isolates. A) MST of isolates from the 2010 cholera outbreak in Haiti. All lines

1063 indicate connections of four or fewer allelic differences. Each node represents a cgST which is

1064 colored by year of isolation. Background shading represent ST designations based on 45 high-

1065 quality SNPs by Katz and colleagues (14). Note that cgST66 contains a mix of color as it

1066 contains both ST1 and ST3. Any isolate from countries other than Haiti is indicated. The length

1067 of the lines is proportional to the number of allelic differences. B) MST constructed from whole

1068 genome SNP data (14). The length of the lines indicates the number of nucleotide substitutions.

1069 Size of the nodes is proportional to the number of isolates. 

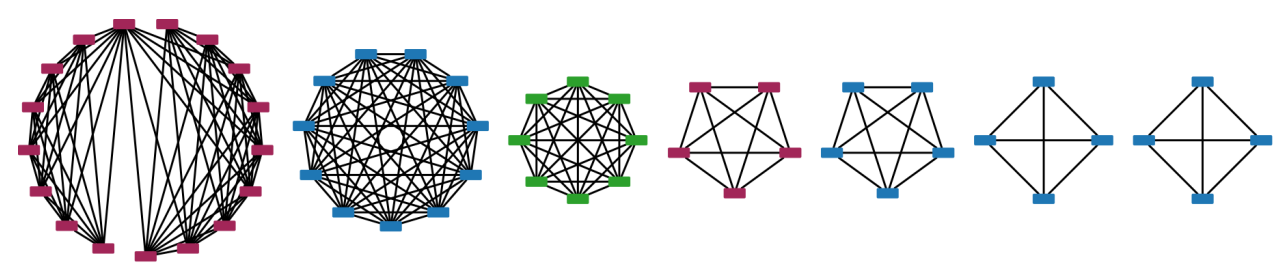

Country of origin
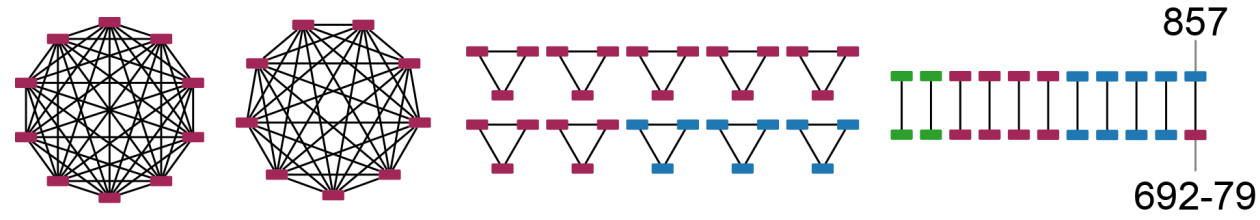

857

$\square$ Bangladesh [65]

$\square$ Brazil [4]

Haiti [23]

$\square$ India [1]

$\square$ Mexico [1]

$\square$ Russia [4]

$\square$ South Korea [1]

$\square$ Sweden [2]

692-79 $\square$ USA [93]

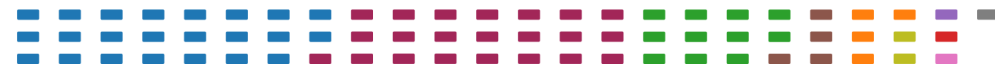

$\square$ Unknown [1]

1071 Fig. 9. Sublineage clusters of non-clinical environmental isolates that are not part of the PG

1072 lineage. Clusters are constructed using networkX (95) and visualized with Cytoscape (96).

1073 Missing loci were assumed to contain the most common allele when calculating allelic

1074 differences. Isolates are connected only if they share 133 allelic differences or fewer with each

1075 other. Each node represents an isolate and is colored by the country of origin. 